Article

\title{
REE Geochemical Characteristic of Apatite: Implications for Ore Genesis of the Zhijin Phosphorite
}

\author{
Liu Xiqiang ${ }^{1,2} \oplus$, Zhang Hui ${ }^{1, *}$, Tang Yong ${ }^{1}$ and Liu Yunlong ${ }^{3}$ \\ 1 Key Laboratory of High-temperature and High-pressure Study of the Earth's Interior Institute of \\ Geochemistry, Chinese Academy of Sciences, Guiyang 550081, China; liuxiqiang@mail.gyig.ac.cn (L.X.); \\ tangyong@mail.gyig.ac.cn (T.Y.) \\ 2 University of Chinese Academy of Sciences, Beijing 100049, China \\ 3 School of Management Science, Guizhou University of Finance and Economics, Guiyang 550025, China; \\ ly1007tt@163.com \\ * Correspondence: zhanghui@mail.gyig.ac.cn
}

Received: 12 October 2020; Accepted: 12 November 2020; Published: 13 November 2020

check for updates

\begin{abstract}
Phosphorite-type rare earth deposits, which are one of the important types of rare earth elements (REE) ore deposits, have attracted increasing attention because of the extreme enrichments in heavy rare earth elements (HREE), including Yttrium (Y). In this study, in situ geochemical analyses of apatite grains from Zhijin phosphorites were conducted using electron probe microanalysis (EMPA) and laser ablation inductively coupled plasma Mass spectrometry (LA-ICP-MS). Based on EPMA Mapping analysis, we show that rare earth elements and Yttrium (REY) entering into the crystal lattice by isomorphism rather than by inclusions of REY-bearing accessory minerals. The post-Archean Australian Shales (PAAS)-normalized REY patterns of the apatite grains are characterized by hat-shaped MREE-enriched patterns. We interpret that this pattern May reflect the REE distribution of seawater at that time. We propose that in a local, reducing environment, dramatically increased the concentration of REY in seawater, and resulted in the MREE-enriched patterns in the ancient ocean. The Main mechanism for the genesis of the Zhijin phosphorite deposit is the apatite crystallizes during the mixing process of REY- and P-rich fluid and oxidizing seawater.
\end{abstract}

Keywords: apatite REY geochemistry; redox; phosphorite genesis; sedimentary phosphorite; Gezhongwu formation

\section{Introduction}

China is a principal producer of the Majority of the REY (rare earth elements and Y) in the world, up to $95 \%$ of the world's demand, and has $136 \mathrm{Mt}$ of REY resources in total, sourced from two types of ore deposits [1]: (1) Bayan Obo carbonatite-type deposits, which provide $80 \%$ of the LREE in the world, but are low in HREE and Y; (2) sedimentary phosphorite-type deposits of South China, which dominate the HREE and Y Market (1.3 million tons (Mt) [2]. In the Guizhou Province, South China, abundant sedimentary phosphorite deposits of the Cryogenian system Doushantuo Stage of and the Terreneuvian Series Meishucunian Stage include plentiful REY and P, a new and important type of REY ore deposit [3-5]. In the Zhijin area, REY resources are Mainly produced from the Meishucunian Stage Gezhongwu Formation. There are 3.5 Mt of REY oxide $\left(\mathrm{REY}_{2} \mathrm{O}_{3}\right)$ resources in phosphate rocks of the Zhijin area, which is highly enriched in $\mathrm{Y}_{2} \mathrm{O}_{3}$ (35.33\%) [4]. Compared with other rare earth deposits with high Th and $U$ concentrations, the Zhijin phosphorite-type rare earth deposit has a lesser environmental impact on mines [2]. Thus development and utilization have far-reaching significance. 
Dating back to the 1960s, three aspects of this type of deposit have been questioned frequently: (1) Is the occurrence of REY due to isomorphic substitution or adsorption? Some studies show that REY enters the lattice of apatite Mainly by isomorphic substitution [2,5-8]. Phase analysis showed that $97 \%$ of the total REY is present in francolite $[9,10]$. Through dissolution experiments of three phosphorites using dilute acid, Gong [9] found identical dissolution efficiency of $\mathrm{P}, \mathrm{Y}$ and Ce for the samples, which inferred that REY is isomorphic in apatite. But there is a lack of direct information about REY's occurrence state. (2) What is the enrichment mechanism for REY in phosphorite deposits? Many researchers have shown that the deviation from Australia's post-Archean average shale normalized REE pattern of modern seawater is a result of several processes, including depositional (e.g., hydrothermal, biological) and post-depositional (e.g., diagenetic, weathering) [2,11-19]. Yet other researchers show the differences in REY patterns are the direct results of redox conditions in ocean chemistry [2,20-27]. (3) What are the sources of P and REY? Do they occur together? Some experts proved that there are several sources of phosphorous: terrestrial sedimentary [3,28], igneous [3], hot spring biologic (e.g., submarine volcanic, [29]) and oceanic (e.g., upwelling current, [11,12]). As one of the two oldest phosphorus-forming events in the earth's history, the early Cambrian phosphorus-forming event is interpreted to be closely related to the dramatic changes of ocean, atmosphere and biological systems during the period $[30,31]$. Evidence showed that these changes were closely related to the breakup of the Rodinia supercontinent [11-13,32-36], which can provide useful information to explain the source of $\mathrm{P}$ and REY.

The focus of this study is to apply novel in situ geochemical analyses of apatite from the phosphorite deposits in the Zhijin region to answer these three outstanding questions. Electron probe microanalysis (EPMA) Mapping will provide information on the occurrence and distribution of REY in apatite grains. Laser ablation inductively coupled plasma Mass spectrometry (LA-ICP-MS) analyses allow us to characterize the enrichment mechanisms and the source of the P and REY. In this study, 27 spots of 20 apatite grains were used for EPMA analysis, and these grains were also used for LA-ICP-MS analysis. The PAAS-normalized REY pattern has long been used as a tool to compare sedimentary rocks [37]. Therefore, all the samples were normalized to PAAS, and the results were compared with those from different sources to determine whether the REY redistribute after deposition. Moreover, the samples were also normalized to seawater, which could provide important information for the interpretation of the distinctive REY patterns. Redox conditions May control the distribution of REY in apatite. $\mathrm{Ce}$ and $\mathrm{Eu}$ are well accepted as a useful tracer for paleo-redox conditions [14,38]. Therefore, we use $\mathrm{Ce} / \mathrm{Ce}^{*}\left(\mathrm{Ce} / \mathrm{Ce}^{*}=\mathrm{Ce}_{\mathrm{N}} /\left(\mathrm{La}_{\mathrm{N}}{ }^{*} \operatorname{Pr}_{\mathrm{N}}\right)^{1 / 2}\right), \mathrm{Eu} / \mathrm{Eu}^{*}\left(\mathrm{Eu} / \mathrm{Eu}^{*}=\mathrm{Eu}_{\mathrm{N}} /\left(\mathrm{Sm}_{\mathrm{N}}{ }^{*} \mathrm{Gd}_{\mathrm{N}}\right)^{1 / 2}\right)$ [37], Y/Ho parameters to restrict the redox environment during apatite formation. These parameters give vital information for us to understand the enrichment mechanism of REY. The average apatite-seawater ( $2500 \mathrm{~m}$ in-depth) REY partition coefficients reveal the correlation of REY between apatite and seawater.

\section{Geologic Background}

The South China Craton lies in the center of Rodinia, between Australia and Laurentian (Figure 1A,B). Rodinia assembled through global orogenic events between $1300 \mathrm{Ma}$ and $900 \mathrm{Ma}$, while the breakup of the supercontinent began at ca. $750 \mathrm{Ma}$ and was not completed until ca. $530 \mathrm{Ma}[34,35]$. Previous studies have shown that Many rift basins within the Yangtze Block are directly related to the breakup of Rodinia [11,34,35]. The Yangtze Block is an important part of the South China Craton (Figure 2A). The two most obvious periods of the Yangtze block drifting out from the breakup of Rodinia are ca. 600-550 Ma and 540 Ma [11]. Many experts [11,39,40] have shown that the breakup of the Rodinia supercontinent affected the carbon dioxide cycle in the earth's atmosphere and hydrosphere and changed the global climate at that time. Two glacial processes (750-700 Ma and 610-575 Ma) controlled the prospering and decaying of the biosphere and also affected the deposition of carbonate, iron, Manganese and phosphorus on the surface of the lithosphere. When the glacial age ended, and the temperature rose, two important biological explosion events occurred (Doushantuo age and Meishucunian age) [11,35]. 


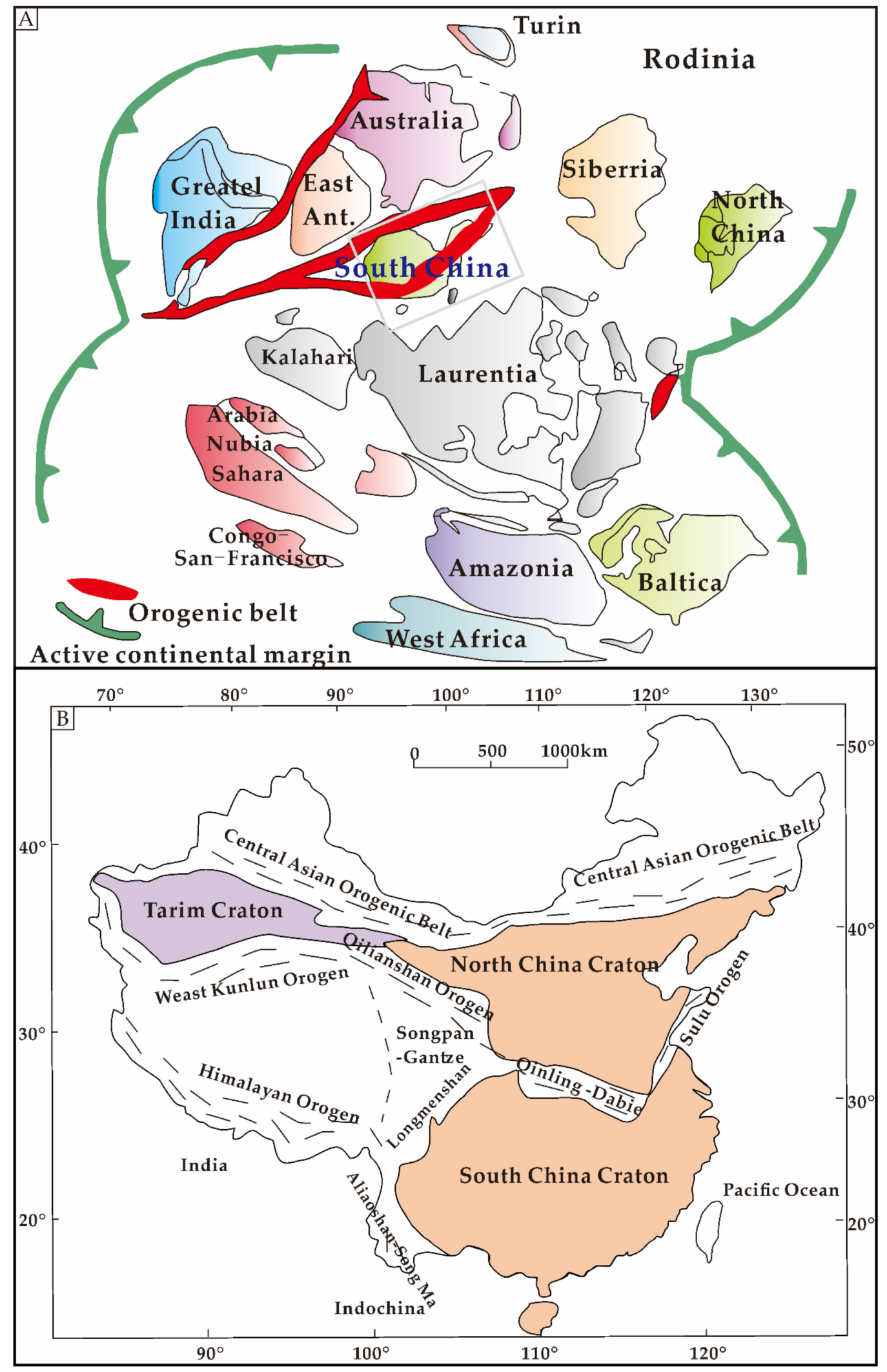

Figure 1. (A) Paleogeographic Map of Rodinia (From Li et al. 2008 [35]); (B) Schematic tectonic Map of China (From Zhao et al. 2012 [36]). 

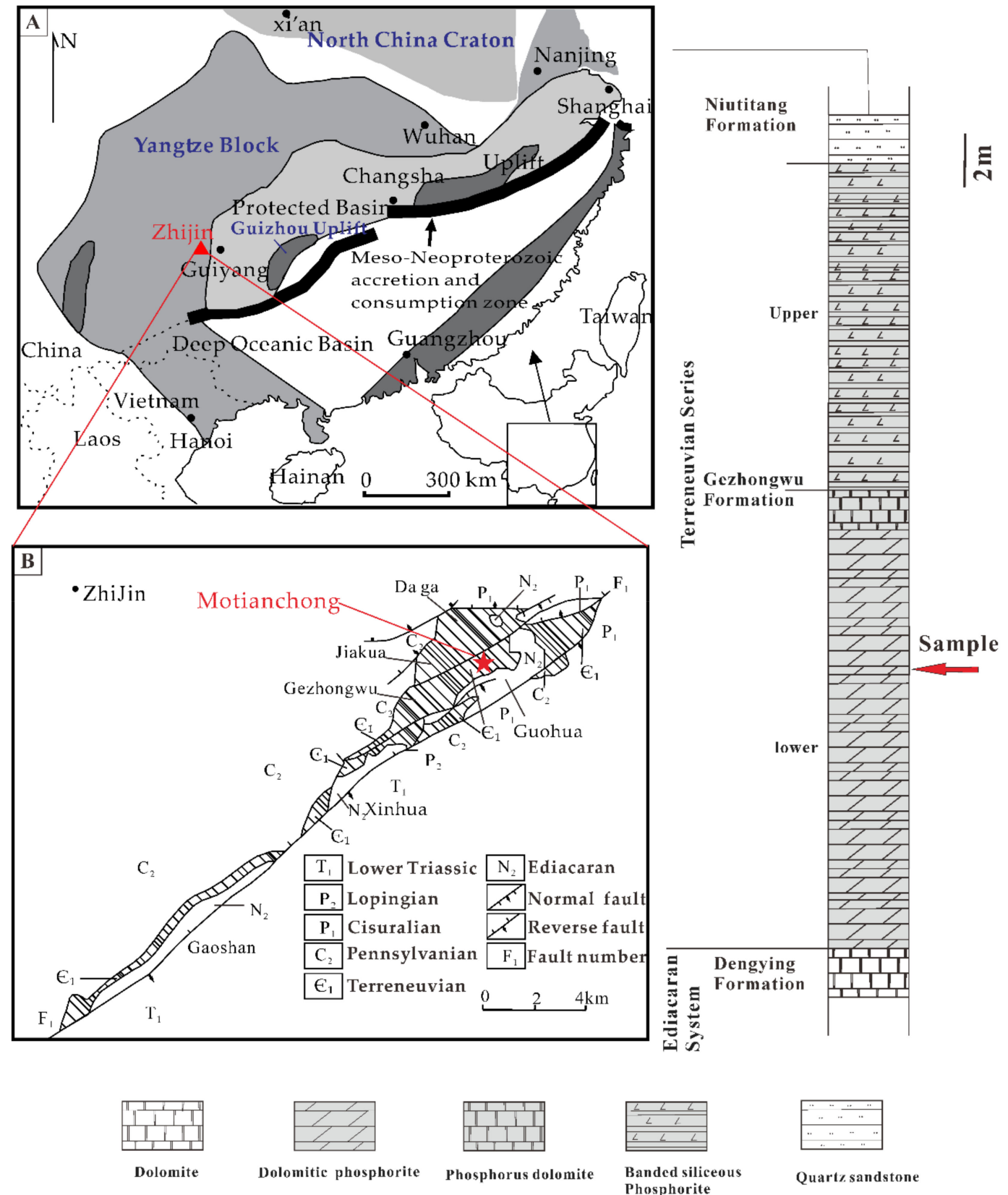

Figure 2. (A) Tectonic Map of the Yangtze Block (From Fan et al. 2016 [30]); (B) Tectonic Map of the Zhijin, Guizhou (From Wu, 1999 [3]); (C) Simplified stratigraphic column of the Gezhongwu Formation at Motianchong ore block.

The Zhijin phosphorite-type REY deposit in Guizhou Province $\left(26^{\circ} 40^{\prime} 24^{\prime \prime} \mathrm{N}\right.$ and $\left.105^{\circ} 51^{\prime} 30^{\prime \prime} \mathrm{E}\right)$ is located $14 \mathrm{~km}$ east of Zhijin County. The width of the ore body is $0.4-4.0 \mathrm{~km}$, and it has an area of $32 \mathrm{~km}^{2}$, extending $20 \mathrm{~km}$ along the NE-SW direction [12]. The occurrence of the REY deposits in the phosphorous-bearing rock series was between the erosion surface of paleo-karst formed by black shale of the Terreneuvian Serious Stage2 Niutitang Formation and dolomite of the Ediacaran system Dengying Formation took place at the bottom of Meishucunian Stage [41]. Structural development Mainly consists of the NE-SW trending Xinhua anticline and a series of normal faults, with locally developed small structures [11,14,42]. These faults are at the southwestern end of the 'central Guizhou uplift' and belong to the western Margin of the Yangtze Block (Figure 2A).

From SW-NE, the Zhijin phosphorite-type REY deposit can be divided into the Gaoshan, Xinhua, Gezhongwu, Jiakua, Guohua and Daga ore blocks (Figure 2B). The distribution of phosphorus and 
REY are Mainly along with the core of Guohua-Gezhongwu anticline and Damachang anticline and are strictly controlled by the strata. From bottom to top, the strata are as follows (Figure 2C): (1) Upper Dengying Formation, which exposes super-thick fine-grained dolomite; (2) Gezhongwu Formation Mainly exposes interbedded phosphorus dolomite and dolomitic phosphorite with thick and thin beds ranging from millimeters to tens of centimeters, which they are the Main ore-bearing beds; (3) Lower Niutitang Formation Mainly develops quartz sandstone.

The intraclastic apatite is Mainly elliptical, with irregular quadrilateral shapes. Particle sizes range from 30 400 $\mu \mathrm{m}$ (Figure 3A-C). They are interpreted as aggregates of microcrystalline carbonate fluorapatite (CFA, shown in the XRD analysis Supplementary Data S1). Hand specimens show that the structural feature is banded with different thickness (Figure 3D). The light-colored band is phosphorous dolomite, and the dark-colored band is of dolomitic phosphorite (Figure 3D). Dolomite and quartz are the Main gangue minerals, which exist as cement around the ores. Dolomitic phosphorite and phosphorus dolomite are Mainly clastic structured, described in detail by previous researchers [5,42]. The rocks have been interpreted to have formed in the sedimentary water, consisting of internal clastic rather than terrigenous clastic particles [42].

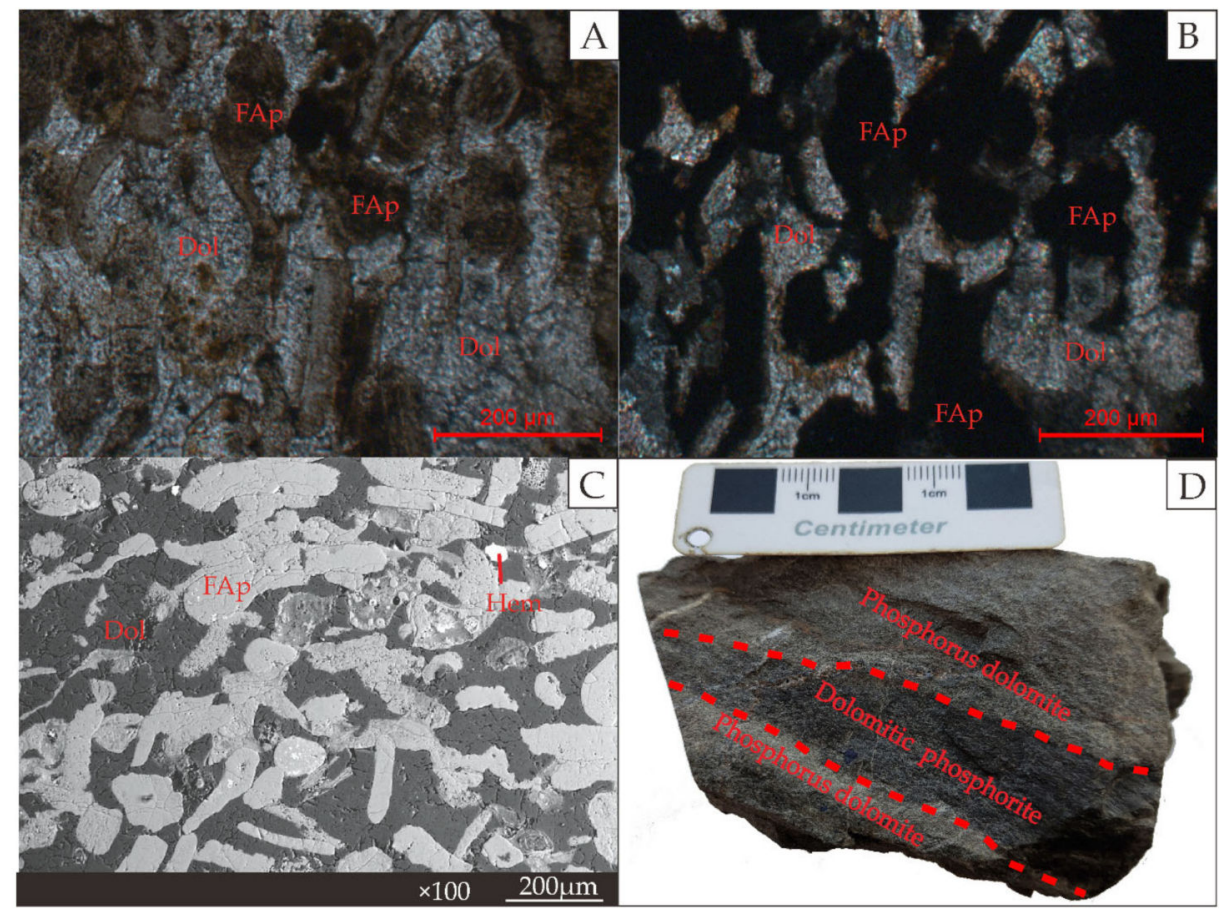

Figure 3. (A) Picture under polarizing microscope (polarized light, MTC-9-5); (B) picture under polarizing microscope (crossed light, MTC-9-5); (C) picture under scanning electron microscope (MTC-9-01); (D) hand specimen (MTC-9-5). FAp-CFA; Hem-hematite; Dol-dolomite.

\section{Samples and Methodology}

In this study, 10 samples of the Motianchong ore block (MTC-1-MTC-10), Mainly consisting of phosphorus-bearing dolomite and dolomitic phosphorite, were collected from the Gezhongwu Formation. There are no obvious morphology differences, so we randomly selected two samples (MTC-7, MTC-9) for single mineral separation and subsequent microanalysis pretreatment. Apatite separation was completed by the Langfang Tuoxuan Rock and Mineral Testing Service Co., Ltd., which adopts conventional gravity separation and Manual selection under a microscope. From the separated apatite grains, 20 grains were selected for EPMA analysis (M1-1-M20-1) and LA-ICP-MS (M1-M20), and 3 of these grains were selected for EPMA Mapping analysis (M9, M11, M20). 
The general chemical formula of apatite is $\mathrm{Ca}_{5}\left(\mathrm{PO}_{4}\right)_{3}(\mathrm{~F}, \mathrm{Cl}, \mathrm{OH})$ [43], where $\mathrm{Z}=\mathrm{F}, \mathrm{Cl}, \mathrm{OH}$. Ca ${ }^{2+}$ can exist in 9-, 7- or 6-fold coordination, and can be substituted by $\mathrm{Sr}^{2+}, \mathrm{Pb}^{2+}, \mathrm{Ba}^{2+}, \mathrm{Mg}^{2+}, \mathrm{Mn}^{2+}$, $\mathrm{Fe}^{2+}, \mathrm{REE}^{3+}, \mathrm{Na}^{+}, \mathrm{K}^{+}$and other cations [44]. $\left(\mathrm{PO}_{4}\right)^{3+}$ can be replaced by $\left(\mathrm{SiO}_{4}\right)^{4-}$, $\left(\mathrm{SO}_{4}\right)^{2-},\left(\mathrm{CO}_{3}\right)^{2-}$ and other complex anions [45]. We calculated concentrations of $\mathrm{OH}$ and $\mathrm{CO}_{2}$ assuming the ideal formula $\mathrm{Ca}_{10} \mathrm{P}_{6} \mathrm{O}_{24} \mathrm{Z}_{2}$, where the cation coefficient at $\mathrm{Ca}, \mathrm{P}$ and $\mathrm{F}$ position are denoted by $\mathrm{X}, \mathrm{Y}, \mathrm{Z}$. The content of $\mathrm{CO}_{2}$ and $\mathrm{OH}$ groups need to be calculated as they are undetectable by the EPMA analytical method. We use the stoichiometric ratio calculation method to calculate the mineral formula with $\mathrm{X}=$ 10. The mineral composition contains $\mathrm{F}$ and $\mathrm{Cl}$, and these anions replace oxygen, so the correction should be done on the oxygen by deducting the content of oxygen replaced by $\mathrm{F}$ and $\mathrm{Cl}$ from the total amount. The deduction formula is: $\mathrm{O}=0.23 \times \mathrm{Cl}$ and $0.42 \times \mathrm{F}$.

Analysis of Major elements in mineral micro-regions was completed in the EPMA Laboratory of Geoscience College of Zhejiang University using a SHIMADZU $1720 \mathrm{H}$ microprobe. The data acquisition time for each element is $30 \mathrm{~s}$ with a beam spot diameter of $5 \mu \mathrm{m}$, accelerating voltage of $15 \mathrm{kV}$, and beam current of $20 \mathrm{nA}$ (For EPMA Mapping analysis, $20 \mathrm{kV}, 100 \mathrm{nA}$, and 10-30 ms/point). The ZAF method was used for correction. The analytical accuracy is $1-2 \%$, and the detection limit is hundreds of ppm. The spectroscopic crystals used are as follows: LSA55(F), RAP (Na, Mg, Al), PET (P, Si, Cl), ADP (S), LIF (Ti, Fe, Ca, Mn). The mineral standard samples used are plagioclase $(\mathrm{Na})$, spodumene $(\mathrm{Al})$, willemite $(\mathrm{Si})$, pyrope $(\mathrm{Mg})$, apatite $(\mathrm{Ca}, \mathrm{P}, \mathrm{F}, \mathrm{S})$, benitoite $(\mathrm{Ti})$, orthoclase $(\mathrm{K})$, almandine $(\mathrm{Fe})$, tugtupite $(\mathrm{Cl})$, rhodonite $(\mathrm{Mn})$.

Some volatile elements such as $\mathrm{F}$ and $\mathrm{Cl}$ in apatite migrate easily with $20 \mathrm{nA}$ beam exposure, so the $\mathrm{F}$ and $\mathrm{Cl}$ contents not accurate. Researchers analyzed the random crystallographic orientations of the apatite, which imply there could have been an increase in F X-ray counts of up to $30-40 \%$ near parallel to the c-axis, while near perpendicular to the c-axis could be quite accurate [46-48], the same as $\mathrm{Cl}$ data. We list the $\mathrm{F}$ and $\mathrm{Cl}$ data in Table 1 (marked $\mathrm{F}^{\mathrm{a}}$ and $\mathrm{Cl}^{\mathrm{a}}$ ) to remind the readers that these measured values, while clearly demonstrate the basic compositional variability and can provide basic calculations for minerals structural formulas, have large uncertainties.

LA-ICP-MS analyses (spot M1-M20) were carried out at the State Key Laboratory of Ore Deposit Geochemistry at the Institute of Geochemistry Chinese Academy of Sciences (Guiyang). The instrument is a GeoLasPro laser ablation system (193 nm, premixed gas, IIIb safety level, $95 \%$ confidence level). The laser spot switch is a Manual version. The laser ablation system is an Agilent 7900 inductively coupled plasma Mass spectrometer. Helium was used as carrier gas, and helium and argon are mixed into the ICP-MS. Spot ablation was carried out using a $44 \mu \mathrm{m}$ spot size at $5 \mathrm{~J} / \mathrm{cm}^{2}$ and $10 \mathrm{~Hz}$ with an $18 \mathrm{~s}$ baseline and $50 \mathrm{~s}$ of ablation. Using NIST610, NIST612, NIST614 and Durago apatite as standard samples, apatite trace element compositions were quantified by using the multi-external standard-internal standard method. The detection limit of REY varies from 0.03-0.4 ppm; the specific values were listed in Liu et al. (2010) [49]. We were careful to avoid analyzing mineral inclusions in apatite. The recommended standard values are from the GeoReM database (http://georem.mpch-main z. gwdg. de/). The off-line processing of analytical data was accomplished using ICPMSDataCal [50,51]. The measured REY isotopes include ${ }^{89} \mathrm{Y},{ }^{139} \mathrm{La},{ }^{140} \mathrm{Ce},{ }^{141} \mathrm{Pr},{ }^{146} \mathrm{Nd},{ }^{147} \mathrm{Sm},{ }^{153} \mathrm{Eu},{ }^{160} \mathrm{Gd},{ }^{159} \mathrm{~Tb},{ }^{163} \mathrm{Dy}$, ${ }^{165} \mathrm{Ho},{ }^{166} \mathrm{Er},{ }^{165} \mathrm{Ho},{ }^{166} \mathrm{Er},{ }^{169} \mathrm{Tm},{ }^{172} \mathrm{Yb}$ and ${ }^{175} \mathrm{Lu}$. 
Table 1. Electron probe microanalysis (EPMA) analytical results for apatite grains (wt.\%).

\begin{tabular}{|c|c|c|c|c|c|c|c|c|c|c|c|c|c|}
\hline Spots & M1-1 & M1-2 & M3-1 & M4-1 & M5-1 & M6-1 & M9-1 & M10-1 & M11-1 & M11-2 & M12-1 & M12-2 & M13-1 \\
\hline $\mathrm{SiO}_{2}$ & 0.03 & 0.02 & 0.02 & 0.03 & 0.01 & 0.06 & 0.05 & 0.04 & 0.01 & 0.08 & 0.18 & 0.05 & 0.09 \\
\hline $\mathrm{Al}_{2} \mathrm{O}_{3}$ & 0.01 & 0.00 & 0.02 & 0.03 & 0.01 & 0.02 & 0.01 & 0.02 & 0.00 & 0.05 & 0.10 & 0.02 & 0.05 \\
\hline $\mathrm{FeO}$ & 0.05 & 0.43 & 0.18 & 0.00 & 0.11 & 0.28 & 0.19 & 0.06 & 0.05 & 0.57 & 0.02 & 0.19 & 0.08 \\
\hline $\mathrm{MnO}$ & 0.00 & 0.04 & 0.02 & 0.01 & 0.05 & 0.04 & 0.00 & 0.01 & 0.01 & 0.00 & 0.00 & 0.03 & 0.03 \\
\hline $\mathrm{MgO}$ & 0.06 & 0.06 & 0.08 & 0.04 & 0.05 & 0.06 & 0.03 & 0.08 & 0.03 & 0.11 & 0.07 & 0.10 & 0.09 \\
\hline $\mathrm{CaO}$ & 55.57 & 56.10 & 55.75 & 56.31 & 56.17 & 55.97 & 54.14 & 55.89 & 56.10 & 53.19 & 55.71 & 55.78 & 55.56 \\
\hline $\mathrm{Na}_{2} \mathrm{O}$ & 0.17 & 0.15 & 0.23 & 0.12 & 0.14 & 0.20 & 0.18 & 0.20 & 0.22 & 0.25 & 0.12 & 0.20 & 0.22 \\
\hline $\mathrm{SO}_{3}$ & 0.22 & 0.39 & 0.10 & 0.34 & 0.32 & 0.59 & 0.31 & 0.35 & 0.49 & 0.24 & 0.39 & 0.08 & 0.26 \\
\hline $\mathrm{P}_{2} \mathrm{O}_{5}$ & 38.17 & 38.77 & 38.43 & 38.42 & 38.62 & 38.62 & 38.73 & 38.02 & 38.66 & 38.19 & 39.16 & 38.90 & 37.78 \\
\hline $\mathrm{F}^{\mathrm{a}}$ & 3.30 & 3.41 & 3.73 & 3.61 & 3.23 & 3.71 & 3.65 & 3.61 & 3.52 & 3.64 & 3.02 & 3.51 & 3.42 \\
\hline $\mathrm{Cl}^{\mathrm{a}}$ & 0.02 & 0.02 & 0.02 & 0.01 & 0.01 & 0.03 & 0.02 & 0.02 & 0.01 & 0.01 & 0.01 & 0.01 & 0.02 \\
\hline $\mathrm{H}_{2} \mathrm{O}^{*}$ & 0.23 & 0.20 & 0.04 & 0.10 & 0.29 & 0.05 & 0.02 & 0.10 & 0.15 & 0.01 & 0.37 & 0.15 & 0.18 \\
\hline $\mathrm{CO}_{2} *$ & 0.64 & 0.62 & 0.66 & 0.65 & 0.63 & 0.59 & 0.37 & 0.69 & 0.60 & 0.40 & 0.42 & 0.59 & 0.69 \\
\hline$-\mathrm{F}=\mathrm{O}$ & 1.38 & 1.43 & 1.57 & 1.52 & 1.36 & 1.56 & 1.53 & 1.51 & 1.48 & 1.53 & 1.27 & 1.48 & 1.44 \\
\hline$-\mathrm{Cl}=\mathrm{O}$ & 0.01 & 0.00 & 0.00 & 0.00 & 0.00 & 0.01 & 0.00 & 0.01 & 0.00 & 0.00 & 0.00 & 0.00 & 0.00 \\
\hline Total & 97.07 & 98.77 & 97.70 & 98.14 & 98.26 & 98.65 & 96.16 & 97.54 & 98.38 & 95.21 & 98.29 & 98.14 & 97.01 \\
\hline $\mathrm{Fe}$ & 0.01 & 0.06 & 0.02 & 0.00 & 0.02 & 0.04 & 0.03 & 0.01 & 0.01 & 0.08 & 0.00 & 0.03 & 0.01 \\
\hline $\mathrm{Mn}$ & 0.00 & 0.01 & 0.00 & 0.00 & 0.01 & 0.01 & 0.00 & 0.00 & 0.00 & 0.00 & 0.00 & 0.00 & 0.00 \\
\hline $\mathrm{Mg}$ & 0.01 & 0.02 & 0.02 & 0.01 & 0.01 & 0.01 & 0.01 & 0.02 & 0.01 & 0.03 & 0.02 & 0.02 & 0.02 \\
\hline $\mathrm{Ca}$ & 9.92 & 9.87 & 9.88 & 9.95 & 9.92 & 9.88 & 9.91 & 9.91 & 9.92 & 9.81 & 9.94 & 9.88 & 9.89 \\
\hline $\mathrm{Na}$ & 0.05 & 0.05 & 0.07 & 0.04 & 0.04 & 0.06 & 0.06 & 0.06 & 0.07 & 0.08 & 0.04 & 0.07 & 0.07 \\
\hline$X$ total & 10 & 10 & 10 & 10 & 10 & 10 & 10 & 10 & 10 & 10 & 10 & 10 & 10 \\
\hline $\mathrm{Si}$ & 0.01 & 0.00 & 0.00 & 0.00 & 0.00 & 0.01 & 0.01 & 0.01 & 0.00 & 0.01 & 0.03 & 0.01 & 0.02 \\
\hline $\mathrm{Al}$ & 0.00 & 0.00 & 0.00 & 0.00 & 0.00 & 0.00 & 0.00 & 0.00 & 0.00 & 0.01 & 0.02 & 0.00 & 0.01 \\
\hline$S$ & 0.03 & 0.05 & 0.01 & 0.04 & 0.04 & 0.07 & 0.04 & 0.04 & 0.06 & 0.03 & 0.05 & 0.01 & 0.03 \\
\hline $\mathrm{P}$ & 5.39 & 5.39 & 5.38 & 5.36 & 5.39 & 5.39 & 5.60 & 5.33 & 5.40 & 5.56 & 5.52 & 5.44 & 5.31 \\
\hline$C^{*}$ & 0.58 & 0.56 & 0.60 & 0.58 & 0.57 & 0.53 & 0.35 & 0.62 & 0.54 & 0.38 & 0.38 & 0.53 & 0.63 \\
\hline$Y$ total & 6 & 6 & 6 & 6 & 6 & 6 & 6 & 6 & 6 & 6 & 6 & 6 & 6 \\
\hline $\mathrm{OH}^{*}$ & 0.26 & 0.22 & 0.04 & 0.11 & 0.31 & 0.06 & 0.02 & 0.11 & 0.16 & 0.02 & 0.41 & 0.16 & 0.20 \\
\hline $\mathrm{F}^{\mathrm{a}}$ & 1.74 & 1.77 & 1.95 & 1.88 & 1.68 & 1.93 & 1.97 & 1.89 & 1.84 & 1.98 & 1.59 & 1.84 & 1.80 \\
\hline $\mathrm{Cl}^{\mathrm{a}}$ & 0.01 & 0.00 & 0.01 & 0.00 & 0.00 & 0.01 & 0.01 & 0.01 & 0.00 & 0.00 & 0.00 & 0.00 & 0.00 \\
\hline $\mathrm{Z}$ total & 2 & 2 & 2 & 2 & 2 & 2 & 2 & 2 & 2 & 2 & 2 & 2 & 2 \\
\hline
\end{tabular}


Table 1. Cont.

\begin{tabular}{|c|c|c|c|c|c|c|c|c|c|c|c|c|c|c|}
\hline Spots & M14-1 & M14-2 & M14-3 & M14-4 & M15-1 & M15-2 & M16-1 & M17-1 & M17-2 & M18-1 & M18-2 & M19-1 & M19-2 & M20-1 \\
\hline $\mathrm{SiO}_{2}$ & 0.04 & 0.04 & 0.02 & 0.05 & 0.01 & 0.01 & 0.03 & 0.08 & 0.05 & 0.03 & 0.01 & 0.04 & 0.05 & 0.04 \\
\hline $\mathrm{Al}_{2} \mathrm{O}_{3}$ & 0.00 & 0.02 & 0.02 & 0.01 & 0.00 & 0.00 & 0.01 & 0.05 & 0.01 & 0.01 & 0.04 & 0.01 & 0.01 & 0.00 \\
\hline $\mathrm{FeO}$ & 0.11 & 0.10 & 0.03 & 0.15 & 0.30 & 0.23 & 0.11 & 0.10 & 0.23 & 0.30 & 0.16 & 0.14 & 0.10 & 0.06 \\
\hline $\mathrm{MnO}$ & 0.00 & 0.02 & 0.00 & 0.01 & 0.00 & 0.04 & 0.03 & 0.01 & 0.00 & 0.00 & 0.00 & 0.01 & 0.00 & 0.02 \\
\hline $\mathrm{MgO}$ & 0.08 & 0.06 & 0.05 & 0.07 & 0.12 & 0.02 & 0.05 & 0.07 & 0.07 & 0.07 & 0.07 & 0.02 & 0.05 & 0.07 \\
\hline $\mathrm{CaO}$ & 56.94 & 56.10 & 56.47 & 54.73 & 55.56 & 56.11 & 57.41 & 56.49 & 53.21 & 55.96 & 54.68 & 53.92 & 56.33 & 58.31 \\
\hline $\mathrm{Na}_{2} \mathrm{O}$ & 0.26 & 0.20 & 0.22 & 0.21 & 0.21 & 0.15 & 0.17 & 0.15 & 0.23 & 0.12 & 0.20 & 0.13 & 0.17 & 0.06 \\
\hline $\mathrm{SO}_{3}$ & 0.16 & 0.16 & 0.35 & 0.31 & 0.37 & 0.43 & 0.2 & 0.38 & 0.26 & 0.29 & 0.14 & 0.65 & 0.31 & 0.13 \\
\hline $\mathrm{P}_{2} \mathrm{O}_{5}$ & 39.40 & 38.13 & 38.40 & 38.68 & 38.35 & 39.42 & 38.98 & 37.99 & 39.03 & 38.30 & 39.15 & 38.54 & 39.22 & 38.04 \\
\hline $\mathrm{F}^{\mathrm{a}}$ & 3.46 & 3.36 & 3.44 & 2.85 & 3.75 & 3.42 & 3.28 & 3.33 & 3.05 & 3.46 & 3.26 & 3.63 & 3.16 & 2.91 \\
\hline $\mathrm{Cl}^{\mathrm{a}}$ & 0.01 & 0.01 & 0.01 & 0.01 & 0.02 & 0.01 & 0.01 & 0.01 & 0.01 & 0.01 & 0.01 & 0.01 & 0.00 & 0.02 \\
\hline $\mathrm{H}_{2} \mathrm{O}^{*}$ & 0.21 & 0.22 & 0.19 & 0.42 & 0.03 & 0.19 & 0.3 & 0.25 & 0.28 & 0.17 & 0.23 & 0.02 & 0.33 & 0.50 \\
\hline $\mathrm{CO}_{2} *$ & 0.64 & 0.72 & 0.70 & 0.46 & 0.63 & 0.49 & 0.74 & 0.74 & 0.25 & 0.67 & 0.41 & 0.32 & 0.55 & 0.98 \\
\hline $\mathrm{F}=\mathrm{O}$ & 1.45 & 1.41 & 1.45 & 1.20 & 1.57 & 1.44 & 1.38 & 1.40 & 1.28 & 1.45 & 1.37 & 1.53 & 1.33 & 1.22 \\
\hline $\mathrm{Cl}=\mathrm{O}$ & 0.00 & 0.00 & 0.00 & 0.00 & 0.01 & 0.00 & 0 & 0.00 & 0.00 & 0.00 & 0.00 & 0.00 & 0.00 & 0.00 \\
\hline Total & 99.84 & 97.73 & 98.44 & 96.77 & 97.76 & 99.09 & 99.92 & 98.25 & 95.38 & 97.93 & 96.99 & 95.91 & 98.95 & 99.91 \\
\hline $\mathrm{Fe}$ & 0.01 & 0.01 & 0.00 & 0.02 & 0.04 & 0.03 & 0.01 & 0.01 & 0.03 & 0.04 & 0.02 & 0.02 & 0.01 & 0.01 \\
\hline $\mathrm{Mn}$ & 0.00 & 0.00 & 0.00 & 0.00 & 0.00 & 0.00 & 0 & 0.00 & 0.00 & 0.00 & 0.00 & 0.00 & 0.00 & 0.00 \\
\hline $\mathrm{Mg}$ & 0.02 & 0.01 & 0.01 & 0.02 & 0.03 & 0.01 & 0.01 & 0.02 & 0.02 & 0.02 & 0.02 & 0.01 & 0.01 & 0.02 \\
\hline $\mathrm{Ca}$ & 9.88 & 9.91 & 9.92 & 9.89 & 9.86 & 9.91 & 9.92 & 9.92 & 9.87 & 9.90 & 9.89 & 9.93 & 9.92 & 9.95 \\
\hline $\mathrm{Na}$ & 0.08 & 0.06 & 0.07 & 0.07 & 0.07 & 0.05 & 0.05 & 0.05 & 0.08 & 0.04 & 0.07 & 0.04 & 0.05 & 0.02 \\
\hline$X$ total & 10 & 10 & 10 & 10 & 10 & 10 & 10 & 10 & 10 & 10 & 10 & 10 & 10 & 10 \\
\hline $\mathrm{Si}$ & 0.01 & 0.01 & 0.00 & 0.01 & 0.00 & 0.00 & 0 & 0.01 & 0.01 & 0.00 & 0.00 & 0.01 & 0.01 & 0.01 \\
\hline $\mathrm{Al}$ & 0.00 & 0.00 & 0.00 & 0.00 & 0.00 & 0.00 & 0 & 0.01 & 0.00 & 0.00 & 0.01 & 0.00 & 0.00 & 0.00 \\
\hline $\mathrm{S}$ & 0.02 & 0.02 & 0.04 & 0.04 & 0.05 & 0.05 & 0.02 & 0.05 & 0.03 & 0.04 & 0.02 & 0.08 & 0.04 & 0.01 \\
\hline $\mathrm{P}$ & 5.40 & 5.32 & 5.33 & 5.52 & 5.38 & 5.50 & 5.32 & 5.27 & 5.72 & 5.35 & 5.60 & 5.61 & 5.46 & 5.13 \\
\hline$C^{*}$ & 0.57 & 0.65 & 0.62 & 0.43 & 0.57 & 0.44 & 0.65 & 0.66 & 0.23 & 0.60 & 0.38 & 0.30 & 0.49 & 0.85 \\
\hline$Y$ total & 6 & 6 & 6 & 6 & 6 & 6 & 6 & 6 & 6 & 6 & 6 & 6 & 6 & 6 \\
\hline $\mathrm{OH}^{*}$ & 0.22 & 0.25 & 0.21 & 0.48 & 0.03 & 0.21 & 0.33 & 0.27 & 0.33 & 0.19 & 0.25 & 0.02 & 0.36 & 0.53 \\
\hline $\mathrm{F}^{\mathrm{a}}$ & 1.77 & 1.75 & 1.78 & 1.52 & 1.96 & 1.78 & 1.67 & 1.73 & 1.67 & 1.81 & 1.74 & 1.98 & 1.64 & 1.46 \\
\hline $\mathrm{Cl}^{\mathrm{a}}$ & 0.00 & 0.00 & 0.00 & 0.00 & 0.01 & 0.00 & 0 & 0.00 & 0.00 & 0.00 & 0.00 & 0.00 & 0.00 & 0.00 \\
\hline $\mathrm{Z}$ total & 2 & 2 & 2 & 2 & 2 & 2 & 2 & 2 & 2 & 2 & 2 & 2 & 2 & 2 \\
\hline
\end{tabular}

${ }^{*}$ represents the calculated value. The analytical number corresponds to the LA-ICP-MS sample number, ${ }^{\text {a }}$ represents inaccuracy. 


\section{Results}

Three grains of EPMA Mapping analysis (Figure 4) show a uniform distribution of $\mathrm{P}$ and $\mathrm{Ca}$ in CFA. From the distribution pictures of $\mathrm{La}, \mathrm{Ce}, \mathrm{Pr}, \mathrm{Nd}, \mathrm{Gd}, \mathrm{Yb}$ and $\mathrm{Y}$, it can be seen REY is evenly distributed in CFA (the whole pictures are shown in Supplementary Data S2).
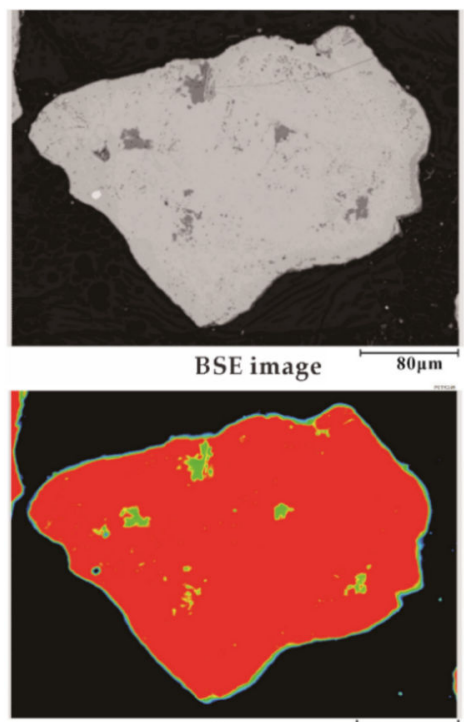

$\mathrm{Ca}$
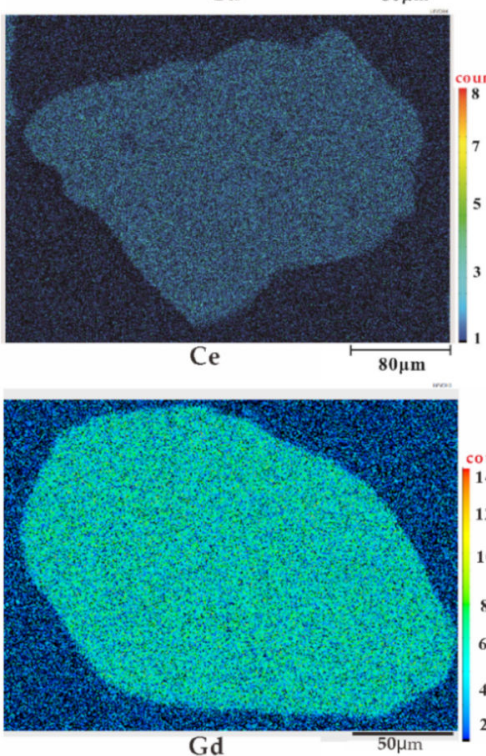

Gd

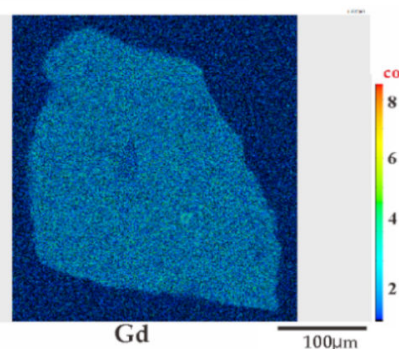

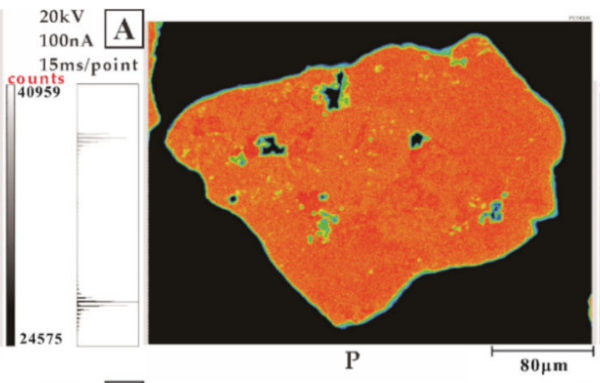
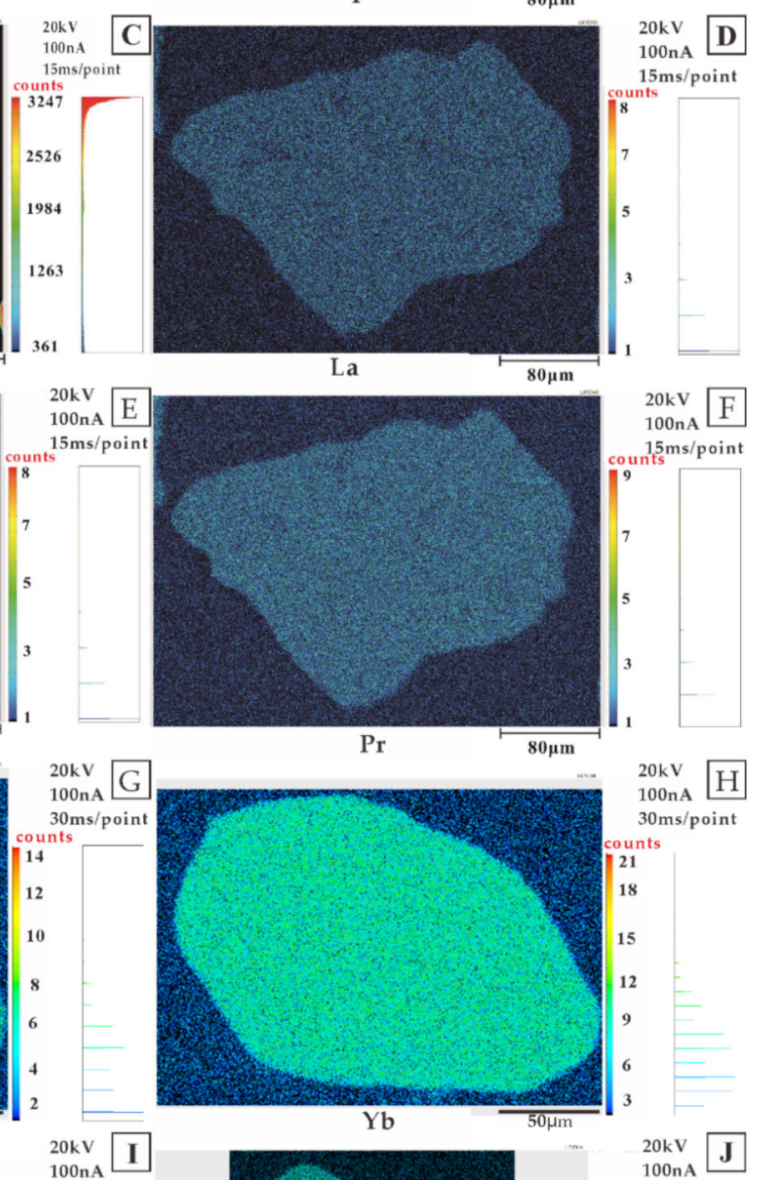

Pr
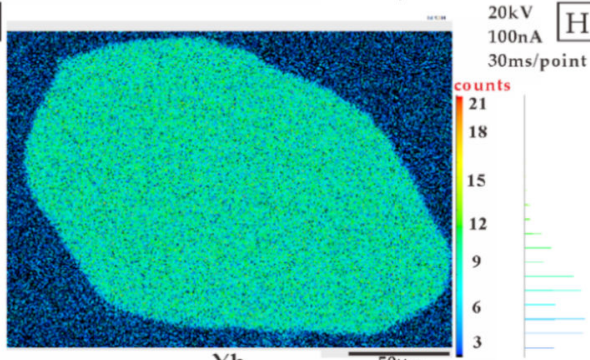

$100 \mathrm{nA}$

$30 \mathrm{~ms} /$ poin

unts

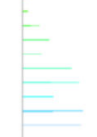

$\mathrm{Yb}$

$20 \mathrm{kV} \mathrm{J}$

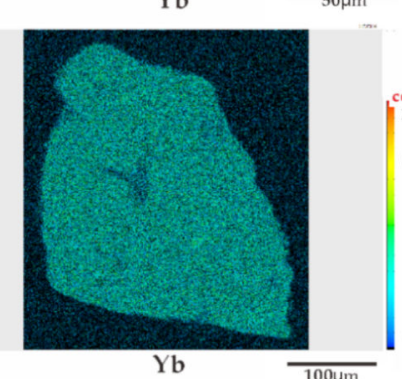

$100 \mathrm{nA}$

$10 \mathrm{~ms} /$ point

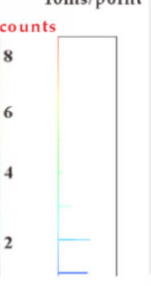

$100 \mu \mathrm{m}$

Figure 4. The EPMA Mapping analysis, (A) Electron back scattered diffraction (BSE) picture of the intraclastic apatite(M20); (B,C) P and Ca Mapping of the intraclastic apatite(M20); (D-F) La, Ce and Pr Mapping of M20; (G,H) Gd, Yb Mapping of M11; (I,J) Gd, Yb Mapping of M9. 
The elemental composition based on the EPMA analysis (Table 1) showed that the content of $\mathrm{CaO}$ and $\mathrm{P}_{2} \mathrm{O}_{5}$ were in the range of 53.19 58.31 wt.\% (average $54.63 \mathrm{wt} . \%$ ) and 38.02 39.42 wt.\% (average $38.60 \mathrm{wt} . \%$ ). Analysis totals ranged from 95.23 99.92 wt.\% (average $97.15 \mathrm{wt} . \%$ ). Although the contents of $\mathrm{F}$ and $\mathrm{Cl}$ are not accurate (up to 40\%) [46], we still give a chemical formula of CFA is as follows: $\left(\mathrm{Fe}_{0-0.08}, \mathrm{Mn}_{0-0.01}, \mathrm{Mg}_{0.01-0.03}, \mathrm{Ca}_{9.81-9.95}, \mathrm{Na}_{0.02-0.08}\right)\left(\mathrm{Si}_{0-0.01}, \mathrm{Al}_{0-0.01}, \mathrm{~S}_{0.01-0.08}, \mathrm{P}_{5.13-5.72}\right.$, $\left.\mathrm{C}_{0.23-0.85}\right)_{6} \mathrm{O}_{24}\left(\mathrm{Cl}_{0-0.01}, \mathrm{~F}_{1.46-1.98 .}, \mathrm{OH}_{0.02-0.53}\right)$.

LA-ICP-MS analyses (Table 2) show the contents of $\Sigma$ REY (the total REE and Y) is 1098 1688 ppm, $\Sigma$ LREE (La-Nd) is 619 932 ppm, $\Sigma$ MREE (Sm-Dy) is 109 154 ppm, the $\Sigma$ HREE (Ho-Lu) is 38.0 53.6 ppm, and $\mathrm{Y}$ is 378 550 ppm, indicating an obvious enrichment of LREE over HREE. The average apatite-seawater $(2500 \mathrm{~m})$ REY partition coefficients (D) are used to reveal the correlation of REY between apatite and seawater. The PAAS-normalized REE patterns for all the samples show a hat-shaped pattern with a slight MREE arch (Figure 5), with negative Ce anomalies and no Eu anomalies. The hat-shaped MREE-enriched patterns are distinct in the studied samples. REY ratios and parameters are listed in Table 3. Ce/Ce* ranges from 0.30 0.32, $\mathrm{Eu} / \mathrm{Eu}^{*}$ is $0.89-0.97, \mathrm{La}_{\mathrm{N}} / \mathrm{Sm}_{\mathrm{N}}$ is 1.18 1.47, $\mathrm{La}_{\mathrm{N}} / \mathrm{Gd}_{\mathrm{N}}$ is $0.81 \sim 0.97, \mathrm{La}_{\mathrm{N}} / \mathrm{Yb}_{\mathrm{N}}$ is $1.68 \sim 2.08, \mathrm{Gd}_{\mathrm{N}} / \mathrm{Yb}_{\mathrm{N}}$ is $1.84 \sim 2.25, \mathrm{Dy}_{\mathrm{N}} / \mathrm{Sm}_{\mathrm{N}}$ is $1.21 \sim 1.30$, and $\mathrm{Y} / \mathrm{Ho}$ is 50.41 57.66. In the PAAS-normalized REE pattern, the La, Gd and Ho are elevated and are followed by a drastic increase of $\mathrm{Pr} \rightarrow \mathrm{Gd}$ (the average $\mathrm{Gd}_{\mathrm{N}} / \mathrm{Pr}_{\mathrm{N}}=1.71$ ), slight decrease of $\mathrm{Gd} \rightarrow \mathrm{Ho}$ (the average $\mathrm{Gd}_{\mathrm{N}} / \mathrm{Ho}_{\mathrm{N}}=1.12$ ) and rapid decrease of $\mathrm{Ho} \rightarrow \mathrm{Lu}$ (the average $\mathrm{Ho}_{\mathrm{N}} / \mathrm{Lu}_{\mathrm{N}}=2.26$ ). The result shows a distinctive feature of the MREE enrichment and HREE depletion in apatite within this study area.

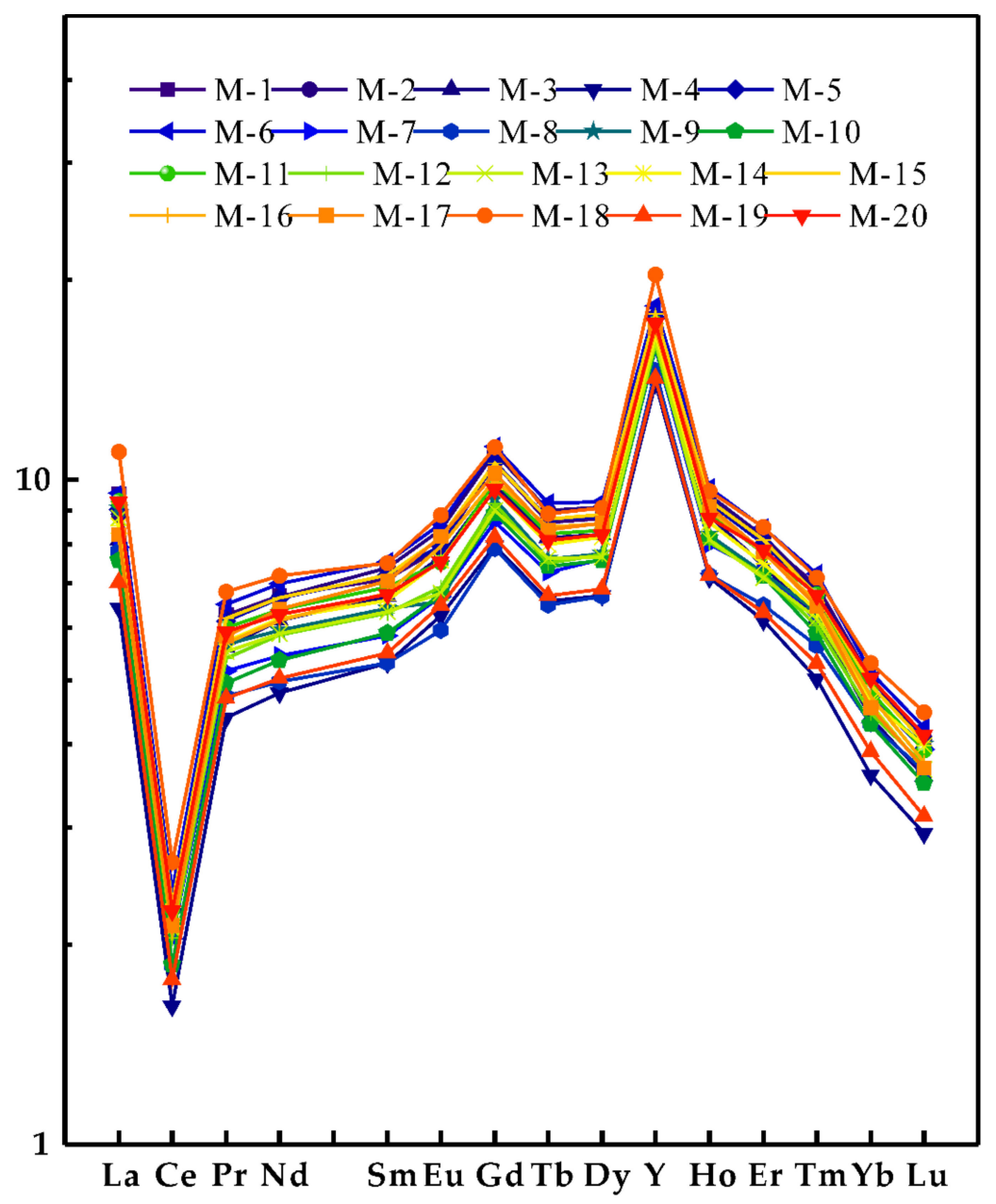

Figure 5. Post-Archean Australian Shales (PAAS)-normalized REY patterns of apatite in this study. 
Table 2. REY contents (in ppm) of the intraclastic apatite grains, determined by laser ablation inductively coupled plasma Mass spectrometry (LA-ICP-MS).

\begin{tabular}{|c|c|c|c|c|c|c|c|c|c|c|c|c|c|c|c|}
\hline Samples & $\mathrm{La}$ & $\mathrm{Ce}$ & Pr & $\mathrm{Nd}$ & $\mathrm{Sm}$ & Eu & Gd & $\mathrm{Tb}$ & Dy & $\mathbf{Y}$ & Ho & Er & $\mathrm{Tm}$ & $\mathrm{Yb}$ & Lu \\
\hline M1 & 364 & 188 & 52.0 & 227 & 39.4 & 8.5 & 49.4 & 6.7 & 41.0 & 472 & 9.3 & 23.2 & 2.8 & 14.2 & 1.8 \\
\hline M2 & 339 & 185 & 51.8 & 226 & 41.0 & 9.1 & 51.3 & 7.0 & 42.6 & 476 & 9.4 & 23.5 & 2.8 & 14.1 & 1.8 \\
\hline M3 & 310 & 164 & 46.5 & 208 & 37.1 & 8.3 & 45.9 & 6.3 & 38.7 & 445 & 8.7 & 21.2 & 2.5 & 12.4 & 1.6 \\
\hline M4 & 245 & 129 & 36.5 & 162 & 29.5 & 6.8 & 37.1 & 5.1 & 31.3 & 378 & 7.1 & 17.5 & 2.0 & 10.1 & 1.3 \\
\hline M5 & 344 & 180 & 50.9 & 225 & 39.9 & 8.6 & 49.6 & 6.8 & 41.2 & 474 & 9.1 & 22.8 & 2.7 & 13.4 & 1.7 \\
\hline M6 & 365 & 192 & 53.9 & 237 & 41.8 & 9.3 & 52.3 & 7.1 & 43.4 & 494 & 9.6 & 24.1 & 2.9 & 14.5 & 1.8 \\
\hline M7 & 303 & 163 & 42.8 & 184 & 32.4 & 7.2 & 40.3 & 5.6 & 35.6 & 426 & 7.9 & 20.7 & 2.5 & 13.3 & 1.7 \\
\hline M8 & 299 & 148 & 39.2 & 169 & 29.4 & 6.4 & 36.7 & 5.0 & 31.3 & 395 & 7.1 & 18.5 & 2.3 & 12.1 & 1.6 \\
\hline M9 & 346 & 176 & 47.0 & 202 & 35.6 & 7.1 & 43.6 & 5.9 & 36.2 & 435 & 8.2 & 20.6 & 2.6 & 13.1 & 1.7 \\
\hline M10 & 2892 & 148 & 41.1 & 182 & 32.7 & 7.3 & 41.4 & 5.7 & 35.5 & 428 & 8.1 & 20.4 & 2.4 & 12.1 & 1.5 \\
\hline M11 & 355 & 180 & 49.8 & 216.1 & 38.3 & 8.1 & 46.5 & 6.4 & 39.3 & 465 & 8.9 & 22.1 & 2.7 & 13.5 & 1.7 \\
\hline M12 & 315 & 163 & 44.7 & 198 & 35.0 & 7.5 & 42.9 & 5.8 & 36.2 & 433 & 8.0 & 20.5 & 2.4 & 12.6 & 1.6 \\
\hline M13 & 335 & 168 & 45.8 & 199 & 35.2 & 7.3 & 41.9 & 5.9 & 35.9 & 438 & 8.1 & 20.3 & 2.5 & 13.0 & 1.6 \\
\hline M14 & 326 & 170 & 47.4 & 210 & 36.6 & 8.2 & 45.1 & 6.2 & 38.4 & 448 & 8.6 & 21.3 & 2.6 & 13.1 & 1.7 \\
\hline M15 & 352 & 183 & 51.4 & 226 & 39.9 & 8.9 & 49.4 & 6.8 & 41.5 & 480 & 9.2 & 23.2 & 2.7 & 13.9 & 1.7 \\
\hline M16 & 313 & 167 & 47.2 & 207 & 37.5 & 8.6 & 46.6 & 6.6 & 40.2 & 460 & 8.9 & 22.2 & 2.6 & 13.3 & 1.6 \\
\hline M17 & 317 & 170 & 48.3 & 217 & 39.1 & 8.9 & 47.7 & 6.5 & 40.3 & 456 & 9.0 & 22.2 & 2.6 & 12.8 & 1.6 \\
\hline M18 & 421 & 211 & 56.4 & 243 & 41.6 & 9.6 & 52.2 & 6.9 & 42.5 & 550 & 9.5 & 24.2 & 2.9 & 15.0 & 1.9 \\
\hline M19 & 268 & 141 & 39.0 & 171 & 30.5 & 7.0 & 38.3 & 5.2 & 32.1 & 383 & 7.1 & 18.0 & 2.1 & 11.0 & 1.3 \\
\hline M20 & 354 & 179 & 49.1 & 213 & 37.4 & 8.1 & 45.2 & 6.3 & 38.8 & 465 & 8.7 & 22.4 & 2.7 & 14.2 & 1.8 \\
\hline PAAS & 38.2 & 79.6 & 8.3 & 33.9 & 5.6 & 1.1 & 4.7 & 0.8 & 4.7 & 27.0 & 9.32 & 23.15 & 2.81 & 14.17 & 1.76 \\
\hline AVG-2500 m & 5.38 & 0.60 & 0.72 & 3.41 & 0.66 & 0.19 & 1.06 & 0.18 & 1.37 & 21.16 & 0.38 & 1.33 & 0.21 & 1.45 & 0.26 \\
\hline AVG-400 m & 2.55 & 0.35 & 0.33 & 1.65 & 0.33 & 0.09 & 0.53 & 0.09 & 0.71 & 10.78 & 0.20 & 0.67 & 0.10 & 0.66 & 0.11 \\
\hline $\mathrm{D} / 10^{6}$ & 65.81 & 296.12 & 68.29 & 62.45 & 56.37 & 43.92 & 42.59 & 34.67 & 28.38 & 21.97 & 22.60 & 16.86 & 13.19 & 9.77 & 7.00 \\
\hline
\end{tabular}

PAAS value (ppm) represents average 23 post-Archean shales from Australia (adapted from Taylor and McLennan [51]; AVG-2500 m and AVG-400 m represent average modern seawater (2500 $\mathrm{m}$ and $400 \mathrm{~m}$ depth) from Alibo et al. [52]; D represents average apatite-seawater (2500 $\mathrm{m})$ REY partition coefficient. 
Table 3. The $\sum$ REY, $\sum$ LREE, $\sum$ MREE and $\sum$ HREE value and the characteristic parameters of REY, from the intraclastic apatite grains.

\begin{tabular}{|c|c|c|c|c|c|c|c|c|c|c|c|c|}
\hline Samples & ¿REY & $\sum$ LREE & ¿MREE & $\sum$ HREE & $\mathrm{La}_{\mathrm{N}} / \mathrm{Gd}_{\mathrm{N}}$ & $\mathrm{La}_{\mathrm{N}} / \mathrm{Yb}_{\mathrm{N}}$ & $\mathrm{Gd}_{\mathrm{N}} / \mathrm{Yb}_{\mathrm{N}}$ & $\mathrm{Ce} / \mathrm{Ce}^{*}$ & $\mathrm{Eu} / \mathrm{Eu}^{*}$ & $\mathrm{Dy}_{\mathrm{N}} / \mathrm{Sm}_{\mathrm{N}}$ & $\mathrm{La}_{\mathrm{N}} / \mathrm{Sm}_{\mathrm{N}}$ & $\mathrm{Y} / \mathrm{Ho}$ \\
\hline M1 & 1500 & 831 & 145 & 51.2 & 0.90 & 1.90 & 2.11 & 0.31 & 0.91 & 1.23 & 1.34 & 50.67 \\
\hline M2 & 1481 & 802 & 151 & 51.6 & 0.81 & 1.77 & 2.19 & 0.31 & 0.93 & 1.23 & 1.20 & 50.61 \\
\hline M3 & 1357 & 728 & 136 & 46.4 & 0.82 & 1.85 & 2.24 & 0.31 & 0.95 & 1.24 & 1.21 & 50.93 \\
\hline M4 & 1098 & 573 & 110 & 38.0 & 0.81 & 1.79 & 2.21 & 0.30 & 0.96 & 1.26 & 1.21 & 53.52 \\
\hline M5 & 1470 & 800 & 146 & 49.8 & 0.85 & 1.90 & 2.24 & 0.30 & 0.91 & 1.22 & 1.25 & 51.80 \\
\hline M6 & 1548 & 8485 & 154 & 53.0 & 0.85 & 1.86 & 2.18 & 0.31 & 0.94 & 1.23 & 1.27 & 51.20 \\
\hline M7 & 1286 & 693 & 121 & 46.1 & 0.92 & 1.68 & 1.84 & 0.32 & 0.94 & 1.30 & 1.36 & 53.67 \\
\hline M8 & 1200 & 654 & 109 & 41.6 & 0.99 & 1.82 & 1.83 & 0.31 & 0.92 & 1.26 & 1.47 & 55.37 \\
\hline M9 & 1380 & 771 & 128 & 46.2 & 0.97 & 1.94 & 2.01 & 0.31 & 0.85 & 1.21 & 1.41 & 53.12 \\
\hline M10 & 1255 & 660 & 123 & 44.5 & 0.85 & 1.76 & 2.07 & 0.30 & 0.93 & 1.29 & 1.29 & 52.86 \\
\hline M11 & 1453 & 801 & 139 & 49.0 & 0.93 & 1.93 & 2.08 & 0.30 & 0.91 & 1.21 & 1.34 & 52.44 \\
\hline M12 & 1326 & 721 & 127 & 45.2 & 0.90 & 1.85 & 2.06 & 0.31 & 0.91 & 1.22 & 1.31 & 54.30 \\
\hline M13 & 1358 & 749 & 126 & 45.4 & 0.97 & 1.91 & 1.96 & 0.30 & 0.89 & 1.21 & 1.38 & 54.37 \\
\hline M14 & 1383 & 753 & 134 & 47.3 & 0.88 & 1.84 & 2.08 & 0.31 & 0.95 & 1.24 & 1.30 & 52.36 \\
\hline M15 & 1490 & 812 & 146 & 50.7 & 0.87 & 1.88 & 2.16 & 0.30 & 0.94 & 1.23 & 1.28 & 52.06 \\
\hline M16 & 1383 & 735 & 140 & 48.7 & 0.82 & 1.73 & 2.12 & 0.31 & 0.97 & 1.27 & 1.21 & 51.57 \\
\hline M17 & 1398 & 752 & 142 & 48.3 & 0.81 & 1.82 & 2.25 & 0.31 & 0.97 & 1.22 & 1.18 & 50.41 \\
\hline M18 & 1688 & 932 & 153 & 53.6 & 0.98 & 2.08 & 2.11 & 0.31 & 0.97 & 1.21 & 1.47 & 57.66 \\
\hline M19 & 1155 & 619 & 113 & 39.6 & 0.85 & 1.80 & 2.11 & 0.31 & 0.97 & 1.25 & 1.28 & 53.77 \\
\hline M20 & 1445 & 795 & 136 & 49.8 & 0.96 & 1.84 & 1.93 & 0.30 & 0.93 & 1.23 & 1.38 & 53.51 \\
\hline AVG & 1383 & 751 & 134 & 47.3 & 0.89 & 1.85 & 2.09 & 0.31 & 0.93 & 1.24 & 1.31 & 52.81 \\
\hline
\end{tabular}

\section{Discussion}

\subsection{Occurrence of REY in Phosphorite}

Previous energy-dispersive $X$-ray spectroscopy (EDS), phase analysis, and experimental dissolution studies have inferred that REY enters the lattice of apatite Mainly by isomorphic substitution $[9,10,53]$. Our LA-ICP-MS signals are constant, which precludes the existence of REY-bearing mineral inclusions that should be characterized by bumps in the spectra. Here, a direct distribution Map of REY is useful to characterize the occurrence of REY in apatite. From the EPMA Mapping analysis, a uniform distribution of the elements $\mathrm{P}$ and $\mathrm{Ca}$ can be clearly seen in Figure 4B-C. The EPMA Mapping analysis of $\mathrm{La}, \mathrm{Ce}, \mathrm{Pr}$, $\mathrm{Nd}, \mathrm{Gd}, \mathrm{Yb}$ and $\mathrm{Y}$ also are displayed in Figure 4. In these graphs, each picture's element has its color scale, so element contents cannot be horizontally compared by color in different pictures. In contrast, in an independent picture, different colors can represent the content of an element. Compared with REY, P and Ca have high counts because they are the Main components of the apatite (Figure 4B,C). Epoxy resins (the surrounding of CFA grains) can be ignored at the high counts of $\mathrm{P}$ and $\mathrm{Ca}$ data, while at the low counts of REY data, epoxy resins have obvious background values. It is a pity that we did not remove the background colors of epoxy resins in REY data, impacting the results. However, we can still recognize the distribution of elements in apatite from colors except for background colors. For example, in Figure $4 \mathrm{H}$, we can see that the distribution of $\mathrm{Yb}$, the green and yellow can represent the relative content of $\mathrm{Yb}$ except for the blue and black background. If $\mathrm{Yb}$ occurs as mineral inclusions, there will be an agglomerate distribution with colors of yellow or green. The same as the other REY, the results showed that $\mathrm{Yb}$ is evenly distributed in the mineral, which indicates REY are associated with isomorphism rather than inclusions of the independent minerals in CFA.

\subsection{Characteristics and Mechanisms REY Patterns}

The enrichment of MREE and the depletion of HREE are important characteristics of the studied samples (Figure 6). Previous studies have intensely debated two Major viewpoints that the geologic processes control REE contents in phosphorites: (1) The MREE-enriched patterns are Mainly caused by other factors during the deposition process or after deposition [13-19]. (2) The differences of REY models are attributed to the direct result of redox conditions in ocean chemistry [20-27]. 


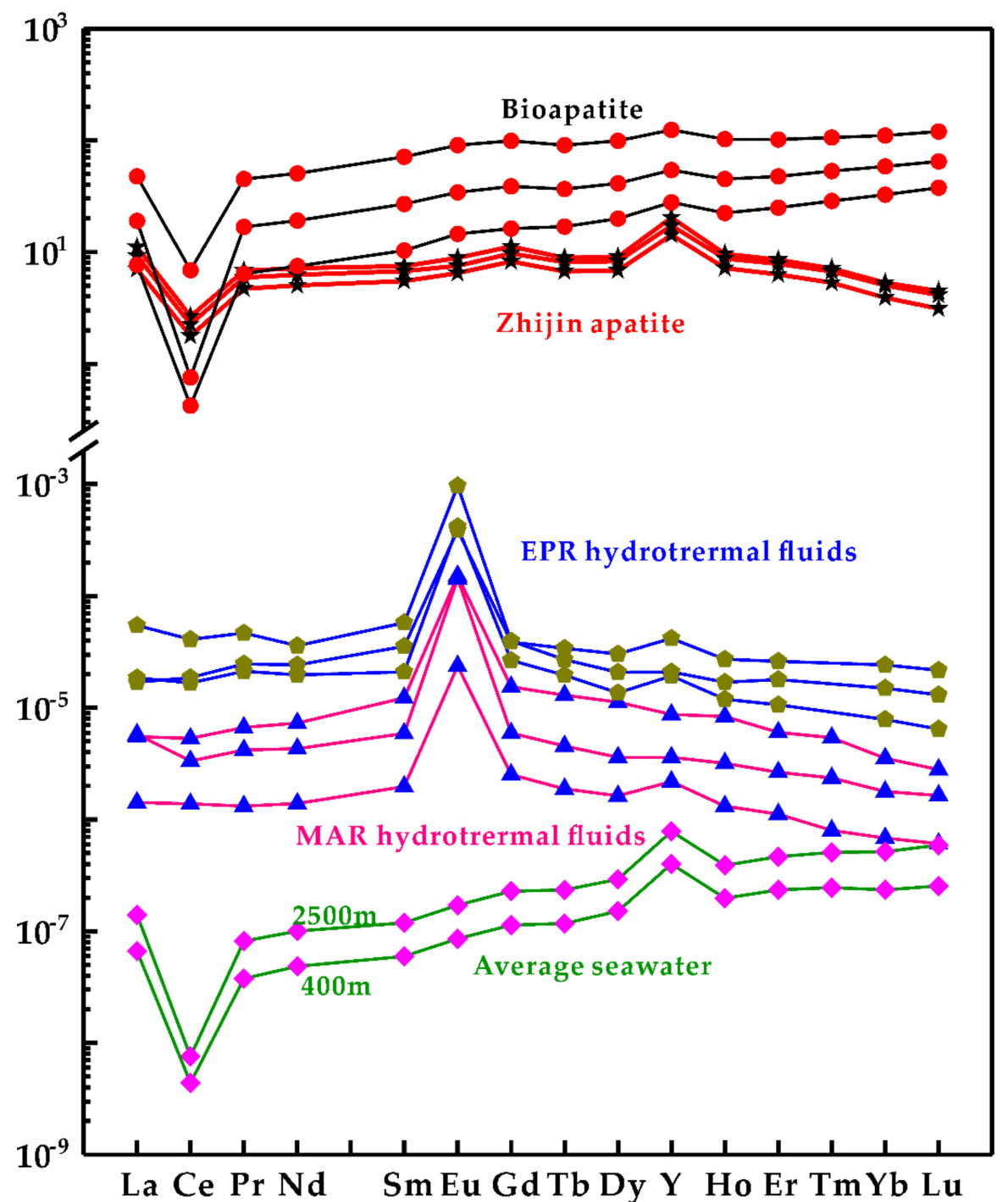

Figure 6. PAAS-normalized REY patterns of different sources: Zhijin apatite; East Pacific Ridge (EPR) hydrothermal systems [19]; hydrothermal fluids from the Mid-Atlantic Ridge (MAR) [15]; average seawater (2500 $\mathrm{m}$ and $400 \mathrm{~m}$ depth, [52]); bioapatite in deep-sea sediments [53].

\subsubsection{Impacts of Depositional or Post-Depositional Processes on REY Patterns}

Suppose REY patterns are owed to depositional or post-depositional processes. There are various variables affecting REY patterns during these processes: (1) Diagenetic inputs-Previous studies $[2,5,18,54]$ shown the original REY characteristics of seawater can be well preserved by fine-grained apatite, and the REY compositions are not easily altered during later burial and diagenesis. Moreover, the influence of burial and diagenesis can be detected by the negative correlation between $\mathrm{Ce} / \mathrm{Ce}^{*}$ and $\mathrm{Dy}_{\mathrm{N}} / \mathrm{Sm}_{\mathrm{N}}[14]$, while the samples in this study do not show this correlation (Figure $7 \mathrm{~A}$ ). (2) Hannigan and Sholkovitz's [17] experiment inferred preferential removal of MREE by the surface water, and in the long-term chemical differentiation process, it will inevitably cause the loss of MREE rather than enrichment. Obviously, aeolian inputs also do not interpret the unique REY patterns in this study. (3) Biological inputs-Previous studies on REY in biological fossils had found that most of the REY patterns in the fossils did not show HREE depletion (Figure 6) [52]. (4) Hydrothermal inputs: In general, $100 \%$ of midocean hydrothermal REE fluxes [2,23] are quickly adsorbed to settling oxyhydroxide particles, which show strong positive $\mathrm{Eu}$ anomalies. Our $\mathrm{Eu} / \mathrm{Eu}^{*}$ data obviously deviate 
from the above values (Figures 6 and 7D), which indicates REY in apatite has no direct relation with hydrothermal inputs.
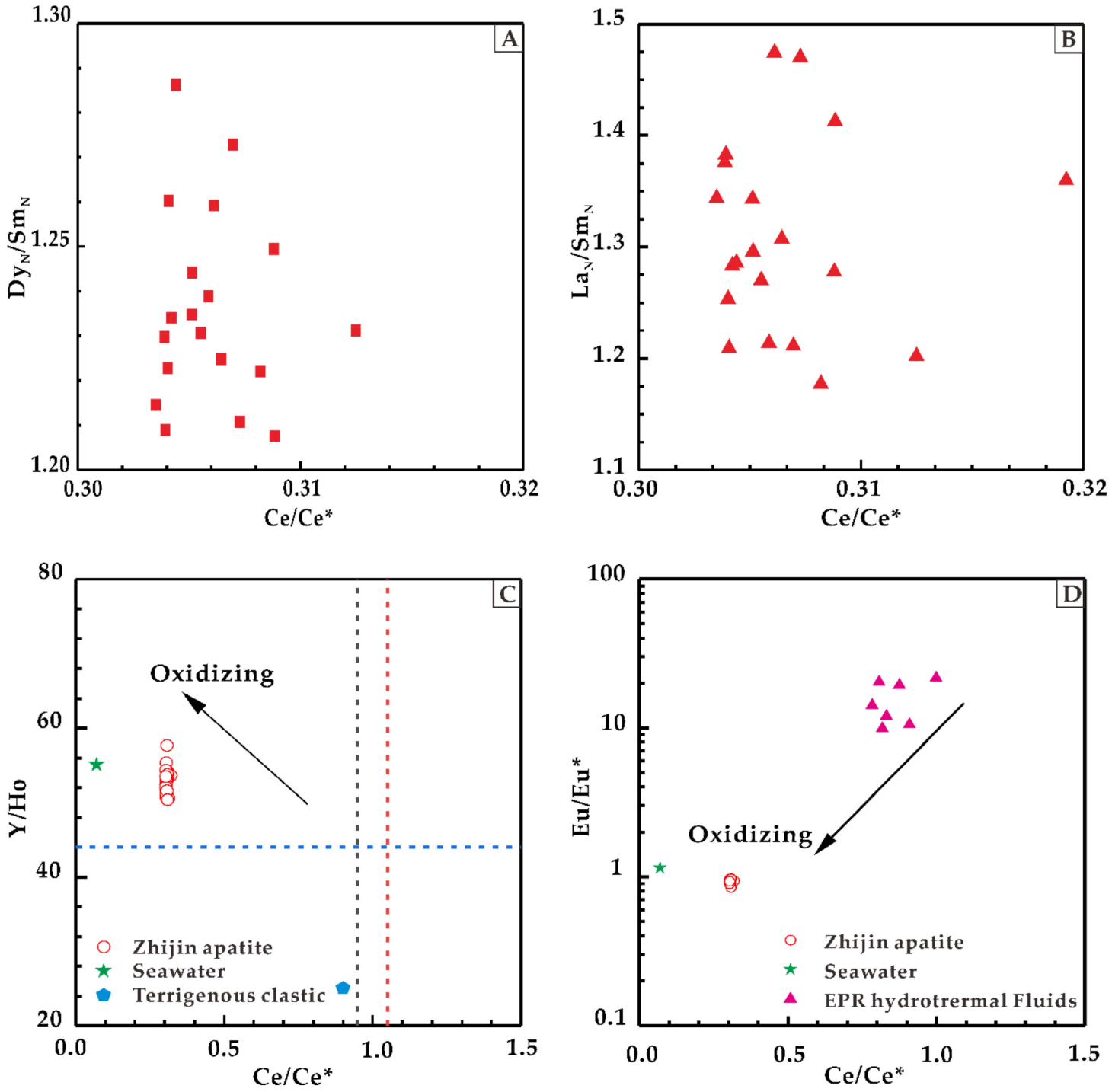

Figure 7. (A) No correlation between $\mathrm{Dy}_{\mathrm{N}} / \mathrm{Sm}_{\mathrm{N}}$ and $\mathrm{Ce} / \mathrm{Ce}^{*} ;(\mathbf{B})$ no correlation between $\mathrm{La}_{\mathrm{N}} / \mathrm{Sm}_{\mathrm{N}}$ and $\mathrm{Ce} / \mathrm{Ce}^{*} ;$ (C) cross-plot of Ce/Ce* vs. Y/Ho; (D) cross-plot of Ce/Ce* vs. Eu/Eu*.

\subsubsection{The source of REY}

Excluding all the above processes, we prefer to favor the deviation of REY patterns due to long-term Marine chemistry variations. In general, the PAAS-normalized REY pattern has long been used as a reliable tool for sedimentary rocks [51,55]. However, Takahashi et al. [56] suggested that for deep-sea sediments, the PAAS normalization was not suitable because different geochemical processes of submarine Materials limited REY. Hence, they adopted a normalized model of deep-sea water [56]. In this study, we use both PAAS (Figure 8) and the deep-sea water model (Figure 9) [52] as standard models to normalize the REY patterns of apatite. We compared PAAS-normalized patterns with seawater-normalized patterns $(2500 \mathrm{~m})$ adopted for the REY in apatite. When normalized to PAAS through comparative analyses, the samples show significant negative Ce anomalies, slight enrichments of LREE, obvious enrichment of MREE and depletions of HREE. While normalized to deep-sea water, the CFA grains display significant positive Ce anomalies, obvious enrichments of LREE, slight enrichments of MREE and clear depletion of HREE. Our REY patterns are similar to 
Emsbo's data [2] of phosphorites (or apatite) from different ages and regions (Figure 8). Furthermore, Emsbo's [2] data are also normalized to seawater (Figure 9) to compare with our patterns. The results showed that the overall curve is smooth from left to right, except $\mathrm{Ce}$, which indicates the single source of REY.

Moreover, previous studies imply the mineral/seawater REY partition coefficients and the logarithm of ionic radius display a simple parabolic relation if REY is a single source [57-59]. According to our result, most data fall on the parabola (Figure 10) (as the Ce value deviates from other elements in Figure 9, it was removed from the scatter diagram). Thus, it also suggests that the REY in apatite is from seawater rather than other contributors.

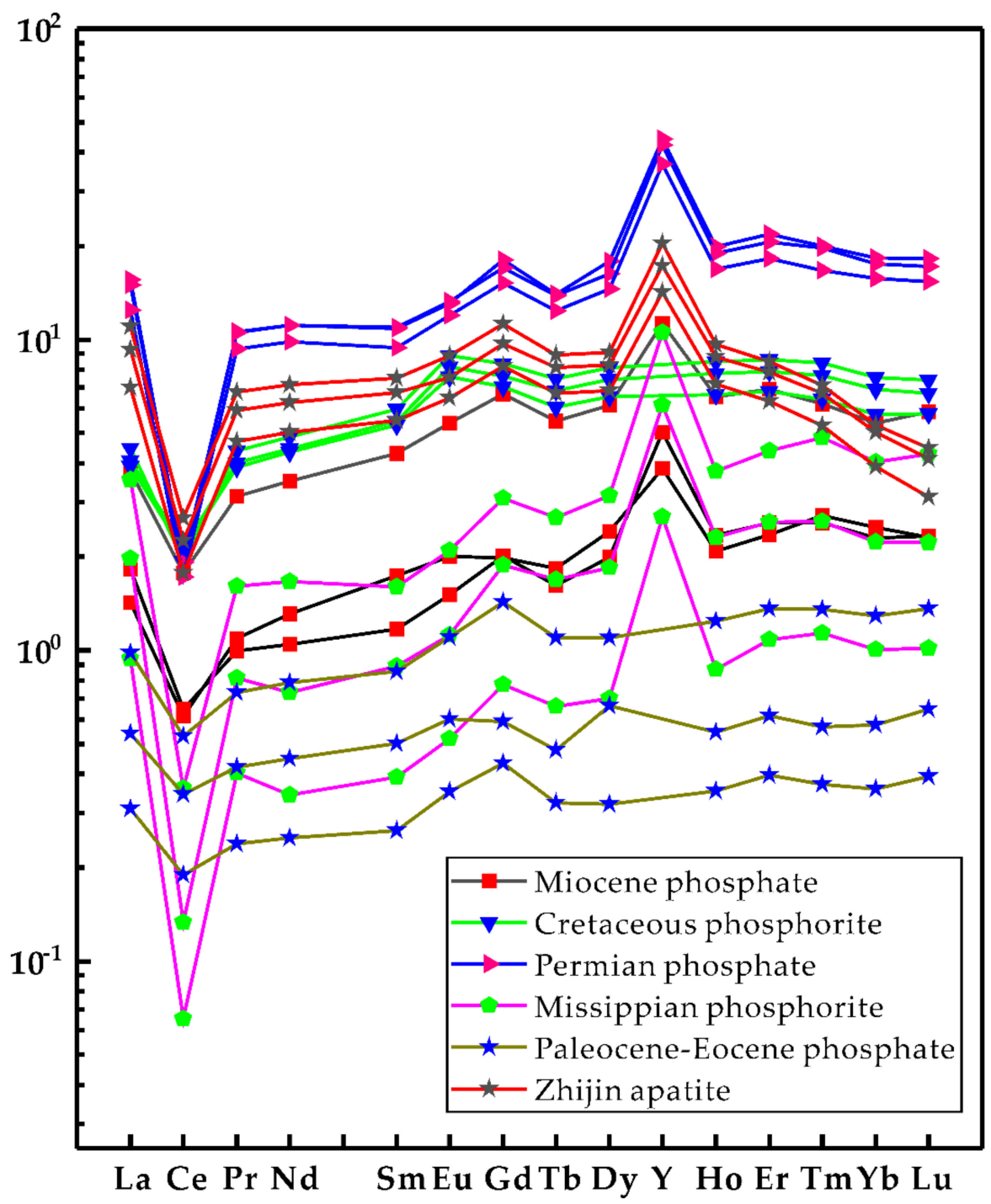

Figure 8. PAAS-normalized REY patterns of the intraclastic apatites of different ages' phosphate or whole rock: Zhijin apatite (study area), Upper Cretaceous phosphorite (Egypt), Upper Permian phosphate (Montana, US.), Miocene phosphate (Florida, US.), Middle Mississippian phosphorite (Alaska, US.), Paleocene-Eocene phosphate (Tunisia) [2]. 


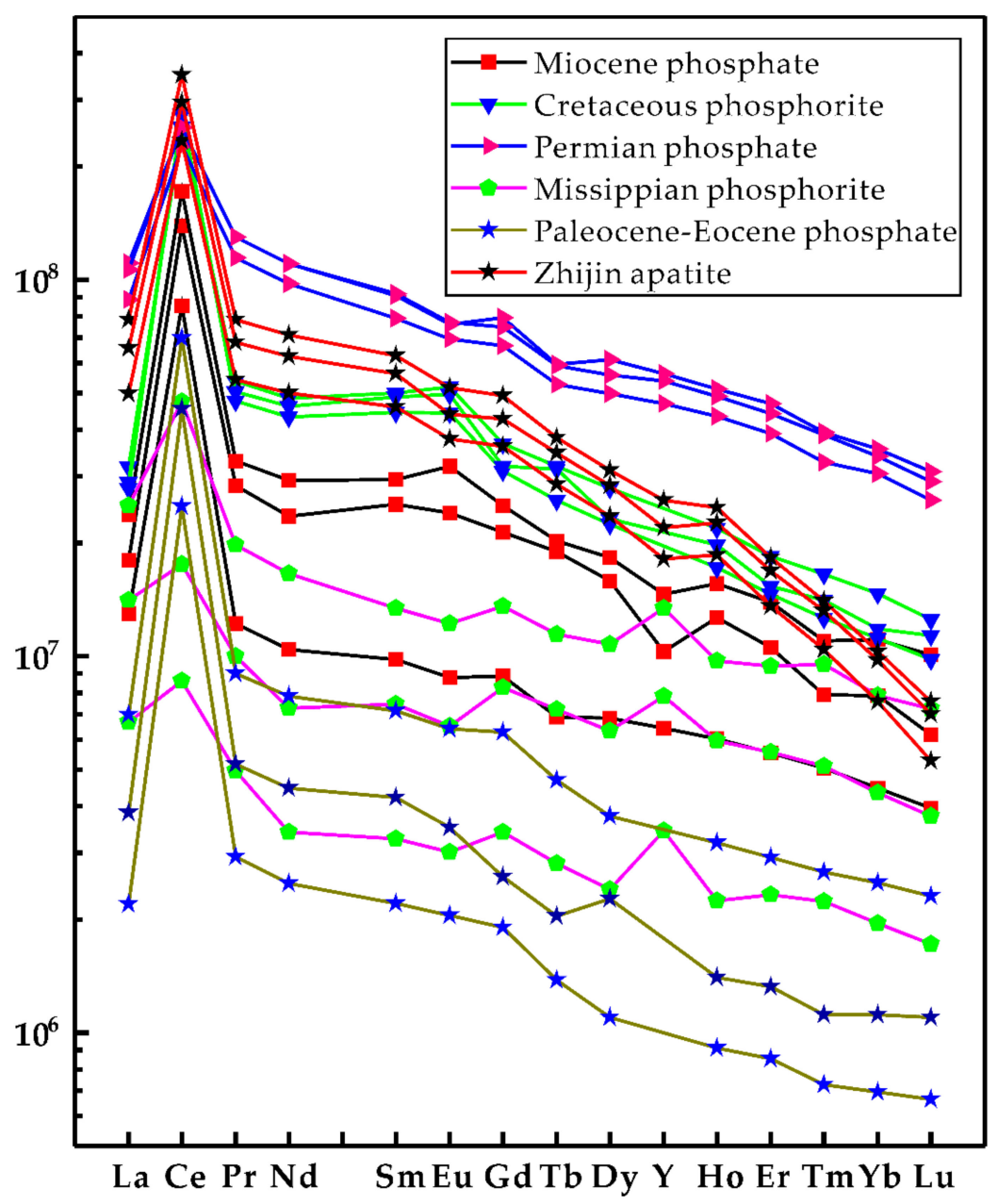

Figure 9. Seawater-normalized REY patterns of the intraclastic apatite of different ages' phosphate or whole rock: Zhijin apatite (study area), Upper Cretaceous phosphorite (Egypt), Upper Permian phosphate (Montana, US.), Miocene phosphate (Florida, US.), Middle Mississippian phosphorite (Alaska, US.), Paleocene-Eocene phosphate (Tunisia) [2].

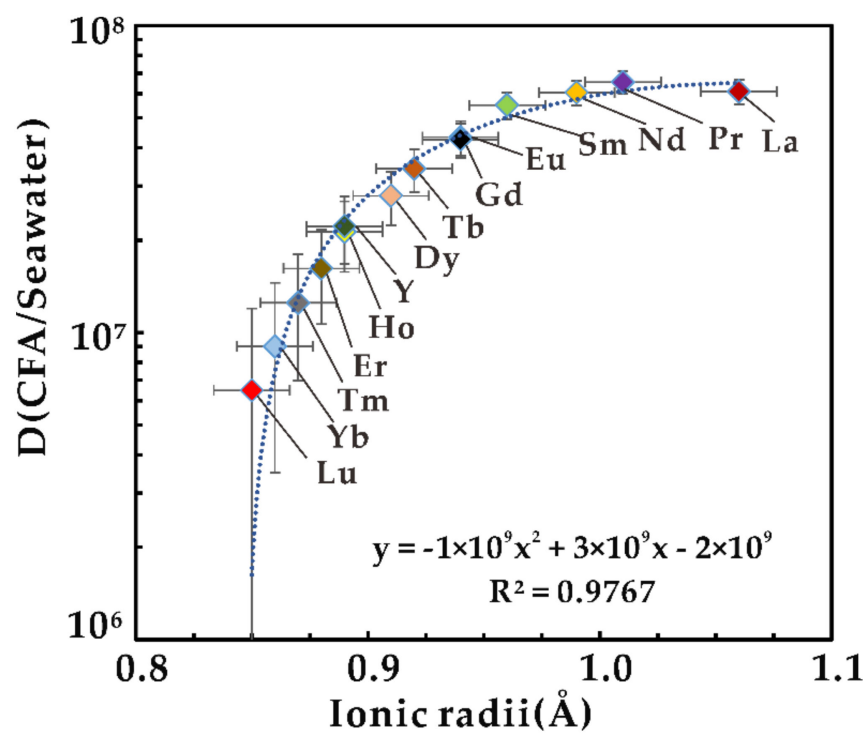

Figure 10. Apatite-seawater REY partition coefficients as a function of ionic radius. 


\subsubsection{Marine Redox Conditions}

Evidence suggests that REY was derived from seawater. Modern seawater is considered to be an oxidizing environment. Hence, it is very important to determine the redox environment when REY enters into apatite. In general, $\mathrm{Ce}, \mathrm{Eu}$ anomalies and $\mathrm{Y} / \mathrm{Ho}$ ratio are considered to restrict the redox environment of paleo-seawater [51,60,61].

1. Ce anomaly: The Ce anomaly of phosphate minerals can represent the paleo-seawater conditions at the time of its formation, only when $\mathrm{La}_{N} / \mathrm{Sm}_{\mathrm{N}}>0.35$, and the $\mathrm{La}_{N} / \mathrm{Sm}_{\mathrm{N}}$ is not related to the $\mathrm{Ce} / \mathrm{Ce}^{*}[62,63]$. In this study, the $\mathrm{La}_{\mathrm{N}} / \mathrm{Sm}_{\mathrm{N}}$ ratio ranges from 1.18 to 1.47 (significantly greater than 0.35 ), and there is no correlation between the $\mathrm{La}_{N} / \mathrm{Sm}_{\mathrm{N}}$ and $\mathrm{Ce} / \mathrm{Ce}^{*}$ (Figure 7B). Hence, the Ce anomaly can be used in determining the paleo-redox environment. When the $\mathrm{Ce} / \mathrm{Ce}^{*}<0.95$, it indicates the oxidizing environment in seawater. On the contrary, the $\mathrm{Ce} / \mathrm{Ce}^{*}>1.05$ interprets the reducing environment. The $\mathrm{Ce} / \mathrm{Ce}^{*}$ value (0.30 0.32) of Zhijin apatite, much less than 1, indicates the deposition of apatite in an oxidizing Marine environment.

2. Y/Ho: In an oxidizing environment, Fe-Mn hydroxides from the seafloor are more likely to adsorb Ho than $Y$, which Makes the adsorption rate for Ho being close to twice that of $Y$, leading to higher $\mathrm{Y} / \mathrm{Ho}$ ratios (44 83) in oxidizing seawater [64,65]. However, Ho will be released from Fe-Mn hydroxides in the reducing environment, resulting in the decrease of $\mathrm{Y} / \mathrm{Ho}$ ratio (reduced to 30) $[15,64,65]$. The inputs of terrigenous clastic Materials (Y/Ho: 23-27) can also lead to low $\mathrm{Y} / \mathrm{Ho}$ values [64]. However, the $\mathrm{Ce} / \mathrm{Ce}^{*}$ of terrigenous clastic Materials can come up to 0.9. Meanwhile, there are no terrigenous clastic particles under the petrographic observation. Our data show a high $\mathrm{Y} / \mathrm{Ho}$ value (ranges from 50.67 54.37, average 52.81), which suggests an oxidizing environment during the formation process of apatite.

3. Eu Anomaly: Under a strongly reducing medium, $\mathrm{Eu}^{3+}$ will be reduced to $\mathrm{Eu}^{2+}$. The redox potential of $\mathrm{Eu}^{3+} / \mathrm{Eu}^{2+}$ decreased significantly at a higher temperature. When the temperature was higher than $200{ }^{\circ} \mathrm{C}, \mathrm{Eu}^{2+}$ became relatively stable. Therefore, $\mathrm{Eu}^{2+}$ is found to be enriched in highly reducing hydrothermal fluids [66]. As the same to $\mathrm{Ce} / \mathrm{Ce}^{*}$, when the $\mathrm{Eu} / \mathrm{Eu}^{*}<0.95$, it indicates the oxidizing environment in seawater. In seawater, however, Eu is not significantly fractionated during the precipitation-dissolution processes (it exists solely in the trivalent state) [14], leading to no obvious $\mathrm{Eu}$ anomaly. Therefore, $\mathrm{Eu} / \mathrm{Eu}^{*}$ anomaly cannot be used to identify the redox environment in seawater but can determine whether REY comes from seawater.

From the cross-plot of $\mathrm{Ce} / \mathrm{Ce}^{*}$ vs. Y/Ho and $\mathrm{Ce} / \mathrm{Ce}^{*}$ vs. Eu/Eu* (Figure $7 \mathrm{C}, \mathrm{D}$ ), all the data in this study fall into the relative oxidizing area, indicate that apatite in the Zhijin phosphorite deposit was formed in an oxidizing environment, which is similar to modern seawater.

\subsubsection{Mechanisms of REY Patterns}

During the Early Cambrian, the surface seawater's oxidation degree and even the whole shallow water area was high, while the deep bottom seawater was anoxic environment [11,12]. Researchers revealed phosphorites in the Yangtze block Mainly deposited at a shallow shelf environment, while no phosphorus deposits were found in the deep-sea environment $[11,12]$. These distributions of deposits indicate that the redox environment restricted the formation of phosphorite. In general, HREE exists by clathrate, and Fe-Mn hydroxides preferentially adsorb LREE and MREE, resulting in the unique REY pattern at oxidizing seawater $[17,20,21]$. The dissolution of adsorbing hydroxides in deep water can locally increase LREE and MREE concentrations by as much as ten times at a reducing environment [22,23]. When these large numbers of LREE and MREE arrived at the upper oxidizing water, due to the change of redox environment, partial Ce and Eu precipitate, leading to the negative $\mathrm{Ce}$ and Eu anomalies, resulting in the distinctive REY patterns.

The crystallization of apatite needs an oxidizing environment, while the release of LREE and MREE requires a reducing environment. Thus, these LREE and MREE need a medium to reach upper oxidizing water. Previous studies have revealed the relationship between the formation of phosphorite and the breakup of the Rodinia supercontinent [11,12]. Sm-Nd and Rb-Sr isochron ages of the Zhijin 
phosphorites are $542 \pm 23 \mathrm{Ma}$ and $541 \pm 12 \mathrm{Ma}$, respectively [11]. The ore-forming ages of apatite coincide with the Yangtze block splitting and drifting from the Rodinia supercontinent, implying the formation of phosphorite deposits was closely related to the breakup events [11,12]. Previous studies $[24-27,67]$ showed that the volcanic activities in the transitional period of Ediacaran-Cambrian might play significant roles in global geological events, and these activities May be responsible for triggering or strengthening the deep-sea reduction degree. Furthermore, these activities accelerated the release of LREE and MREE from Fe-Mn hydroxides, heated the deep seawater and formed ocean currents and columns. Carrying the large numbers of LREE and MREE, the currents and columns upwelled to upper oxidizing water, leading to distinctive REY patterns of CFA.

\subsection{The Source of $P$ and the Diagenesis Mechanism}

$\mathrm{Nd}$ isotopic model ages $\left(\mathrm{t}_{\mathrm{DM}}\right)$ can be used to trace the sediment source, and the variation of $\mathrm{Nd}$ model age $\left(\varepsilon_{\mathrm{Nd}}(\mathrm{t})\right)$ is a reliable tracer of Mantle-derived Materials [68]. The $t_{\mathrm{DM}}$ of the Zhijin phosphorite deposit (1453-1477 Ma) is obviously lower than that of around seawater in Cambrian, and the $\varepsilon_{\mathrm{Nd}}(\mathrm{t})(-2.37 \sim-2.65)$ is higher than that of seawater during the Cambrian period, which suggests that the initial Material for large-scale phosphorous mineralization May be Mainly derived from the Mantle, and also indicates the interaction between crust and Mantle is strong during the breakup of Rodinia. Liu and Shi [11,12] inferred that the breakup of the Rodinia supercontinent led to the upwelling of the hydrothermal fluids, which carried a large number of P-rich Materials from the Mantle. When the P-rich fluid overflowed into deep-sea water, due to the crystallization of apatite requires an oxidizing environment, these P-rich bearing and REY would not precipitate but floated and upwelled with currents to shallow oxidizing seawater. Other researchers suggested Fe-Mn hydroxides could efficiently adsorb not only REY but also P and then sank into the seafloor sediments, which is the Main mechanism of dissolved phosphorus and REY entering the seafloor sediments [69,70]. No Matter P-rich Materials were from the Mantle or the release of Fe-Mn hydroxides, the upwelling P-rich bearings provided abundant Materials for the crystallization of apatite.

Therefore, the P and released LREE and MREE upwelled to upper shallow water with the ocean currents and columns. Due to the change of redox environment, these P-rich and REY Materials crystalized to apatite, resulting in the distinctive REY patterns in the Zhijin phosphorite deposit.

\section{Conclusions}

1. Based on the EPMA Mapping analysis, it suggested that the REY occurring in the Zhijin phosphorite originate from isomorphism rather than mineral inclusions in the CFA;

2. There is a simple parabolic relationship between the mineral/seawater REE partition coefficients and the ionic radii, so to a certain extent, it implies that the REY in apatite is originated from seawater rather than other sources;

3. In a local reducing environment, the released REY from hydroxides dramatically increases the concentration of REY in the ocean seawater;

4. The breakup of the supercontinent leads to the upwelling of the hydrothermal fluids, which was carrying abundant P-rich Materials. These upwelling processes will enhance or lead to the reducing environment and dissolution of considerable LREEs and MREEs. When the P-rich fluid and REY reach the upper oxidizing water with the upwelling currents to upper oxidizing shallow water, REY-rich apatite formed during this mixing process.

Supplementary Materials: The following are available online at http://www.mdpi.com/2075-163X/10/11/1012/s1, Supplementary Data S1; Supplementary Data S2.

Author Contributions: Conceptualization, L.X. and Z.H.; methodology, T.Y. and L.Y.; software, L.Y.; formal analysis, L.X.; investigation, Z.H. and L.X.; writing—original draft preparation, L.X.; writing—review and editing, L.X. and Z.H. All authors have read and agreed to the published version of the manuscript. 
Funding: This work was financially supported by the Public Beneficial, Basic Geological Project of Department of Land and Resources of Guizhou Province (2016-09-1).

Conflicts of Interest: The authors declare no conflict of interest.

\section{References}

1. Long, K.R.; Van Gosen, B.S.; Foley, N.K.; Cordier, D. The Principal Rare Earth Elements Deposits of the United States: A Summary of Domestic Deposits and a Global Perspective. In Non-Renewable Resource Issues; Springer: Dordrecht, The Netherlands, 2012; pp. 131-155. [CrossRef]

2. Emsbo, P.; McLaughlin, P.I.; Breit, G.N.; Du Bray, E.A.; Koenig, A.E. Rare earth elements in sedimentary phosphate deposits: Solution to the global REE crisis? Gondwana Res. 2015, 27, 776-785. [CrossRef]

3. Wu, X.H. Phosphorites in Guizhou; Geological Publishing House: Beijing, China, 1999; pp. 1-115. (In Chinese)

4. Meng, Q.T.; Lan, A.P.; Ye, C. A Report on Integrated Exploration of Phosphorus (Rare Earth) Deposits in Zhijin Area, Guizhou Province. Fourth Geological Brigade of Guizhou Geological and Mineral Exploration and Development Bureau: Guizhou, China, 2014. (In Chinese)

5. Zhu, B.; Jiang, S.-Y. A LA-ICP-MS analysis of rare earth elements on phosphatic grains of the Ediacaran Doushantuo phosphorite at Weng'an, South China: Implication for depositional conditions and diagenetic processes. Geol. Mag. 2017, 154, 1381-1397. [CrossRef]

6. Paul, S.A.; Volz, J.B.; Bau, M.; Köster, M.; Kasten, S.; Koschinsky, A. Calcium phosphate control of REY patterns of siliceous-ooze-rich deep-sea sediments from the central equatorial Pacific. Geochim. Cosmochim. Acta 2019, 251, 56-72. [CrossRef]

7. Joosu, L.; Lepland, A.; Kirsimäe, K.; Romashkin, A.E.; Roberts, N.M.; Martin, A.P.; Črne, A.E. The REE-composition and petrography of apatite in 2Ga Zaonega Formation, Russia: The environmental setting for phosphogenesis. Chem. Geol. 2015, 395, 88-107. [CrossRef]

8. Hughes, J.M.; Rakovan, J.F. Structurally Robust, Chemically Diverse: Apatite and Apatite Supergroup Minerals. Elements 2015, 11, 165-170. [CrossRef]

9. Gong, M.L. Phase Analysis and Geological Prospecting, 2nd ed.; Geological Publishing House: Beijing, China, 2007; pp. 161-172. (In Chinese)

10. Chen, J.Y.; Zhang, J.; Yang, R.D. Mode of occurrence of rare earth elements in phosphorite in Zhijin county, Guizhou Province, China. Acta Mineral. Sin. 2010, 30, 123-129, (In Chinese with English abstract). [CrossRef]

11. Shi, C.H. Formation of phosphorite deposit, Breakup of Rodinia Supercontinent and Biology Explosion-A Case Study of Weng'an, Kaiyang and Zhijin Phosphorite Deposits of Guizhou Province. Ph.D. Thesis, Institute of Geochemistry Chinese Academy of Sciences, Guiyang, China, 2005. (In Chinese).

12. Liu, S.R. Study of the Mineralization Process of Zhijin Xinhua Phosphate Deposit Using Microbeam Analyses. Ph.D. Thesis, Institute of Geochemistry Chinese Academy of Sciences, Guiyang, China, 2008. (In Chinese).

13. McArthur, J.; Walsh, J. Rare-earth geochemistry of phosphorites. Chem. Geol. 1984, 47, 191-220. [CrossRef]

14. Shields, G.A.; Stille, P. Diagenetic constraints on the use of cerium anomalies as palaeoseawater redox proxies: An isotopic and REE study of Cambrian phosphorites. Chem. Geol. 2001, 175, 29-48. [CrossRef]

15. Bau, M.; Dulski, P. Comparing yttrium and rare earths in hydrothermal fluids from the Mid-Atlantic Ridge: Implications for $\mathrm{Y}$ and REE behaviour during near-vent mixing and for the $\mathrm{Y} / \mathrm{Ho}$ ratio of Proterozoic seawater. Chem. Geol. 1999, 155, 77-90. [CrossRef]

16. Sholkovitz, E.R.; Landing, W.M.; Lewis, B.L. Ocean particle chemistry: The fractionation of rare earth elements between suspended particles and seawater. Geochim. Cosmochim. Acta 1994, 58, 1567-1579. [CrossRef]

17. Hannigan, R.E.; Sholkovitz, E.R. The development of middle rare earth element enrichments in freshwaters: Weathering of phosphate minerals. Chem. Geol. 2001, 175, 495-508. [CrossRef]

18. Martin, E.E.; Scher, H.D. Preservation of seawater Sr and Nd isotopes in fossil fish teeth: Bad news and good news. Earth Planet. Sci. Lett. 2004, 220, 25-39. [CrossRef]

19. Douville, E.; Bienvenu, P.; Charlou, J.L.; Donval, J.P.; Fouquet, Y.; Appriou, P.; Gamo, T. Yttrium and rare earth elements in fluids from various deep-sea hydrothermal systems. Geochim. Cosmochim. Acta 1999, 63, 627-643. [CrossRef]

20. Elderfield, H.; Greaves, M.J. The rare earth elements in seawater. Nat. Cell Biol. 1982, 296, 214-219. [CrossRef] 
21. Jiang, S.-Y.; Zhao, H.; Chen, Y.-Q.; Yang, T.; Yang, J.-H.; Ling, H.-F. Trace and rare earth element geochemistry of phosphate nodules from the lower Cambrian black shale sequence in the Mufu Mountain of Nanjing, Jiangsu province, China. Chem. Geol. 2007, 244, 584-604. [CrossRef]

22. De Baar, H.J.; Bacon, M.P.; Brewer, P.G.; Bruland, K.W. Rare earth elements in the Pacific and Atlantic Oceans. Geochim. Cosmochim. Acta 1985, 49, 1943-1959. [CrossRef]

23. Elderfield, H.; Upstill-Goddard, R.; Sholkovitz, E. The rare earth elements in rivers, estuaries, and coastal seas and their significance to the composition of ocean waters. Geochim. Cosmochim. Acta 1990, 54, 971-991. [CrossRef]

24. Jenkyns, H.C. Geochemistry of oceanic anoxic events. Geochem. Geophys. Geosyst. 2010, 11. [CrossRef]

25. Gill, B.; Lyons, T.W.; Young, S.A.; Kump, L.R.; Knoll, A.H.; Saltzman, M.R. Geochemical evidence for widespread euxinia in the Later Cambrian ocean. Nat. Cell Biol. 2011, 469, 80-83. [CrossRef]

26. McLaughlin, P.I.; Emsbo, P.; Brett, C.E. Beyond black shales: The sedimentary and stable isotope records of oceanic anoxic events in a dominantly oxic basin (Silurian; Appalachian Basin, USA). Palaeogeogr. Palaeoclim. Palaeoecol. 2012, 367-368. [CrossRef]

27. Harper, D.A.T.; Hammarlund, E.U.; Rasmussen, C.M.Ø. End Ordovician extinctions: A coincidence of causes. Gondwana Res. 2014, 25, 1294-1307. [CrossRef]

28. Zhang, J.; Sun, C.M.; Gong, M.L. Geochemical Characteristics and Occurrence States of the REE Elements of the Phosphorite in Xinhua, Zhijin, Guizhou. Chin. Rare Earths 2007, 28, 75-79, (In Chinese with English abstract). [CrossRef]

29. Yang, W.D.; Xiao, J.K.; Chen, F. Sedimentology, Geochemistry and Sustainable Development Strategy of Phosphorite in Yunnan and Guizhou; Geological Publishing House: Beijing, China, 1997; pp. 9-14. (In Chinese)

30. Fan, H.; Wen, H.; Zhu, X. Marine redox conditions in the Early Cambrian ocean: Insights from the Lower Cambrian phosphorite deposits, South China. J. Earth Sci. 2016, 27, 282-296. [CrossRef]

31. Qiao, W.; Lang, X.; Peng, Y.; Jiang, K.; Chen, W.; Huang, K.; Shen, B. Sulfur and oxygen isotopes of sulfate extracted from Early Cambrian phosphorite nodules: Implications for Marine redox evolution in the Yangtze Platform. J. Earth Sci. 2016, 27, 170-179. [CrossRef]

32. Li, Z.X.; Zhang, L.; Powell, C.M. Positions of the East Asian cratons in the Neoproterozoic supercontinent Rodinia. J. Geol. Soc. Aust. 1996, 43, 593-604. [CrossRef]

33. Powell, C.M.; Li, Z.-X.; McElhinny, M.W.; Meert, J.G.; Park, J.K. Paleomagnetic constraints on timing of the Neoproterozoic breakup of Rodinia and the Cambrian formation of Gondwana. Geology 1993, 21, 889-892. [CrossRef]

34. Wang, J. History of Neoproterozoic rift basins in South China: Implications for Rodinia break-up. Precambrian Res. 2003, 122, 141-158. [CrossRef]

35. Li, Z.; Bogdanova, S.; Collins, A.; Davidson, A.; De Waele, B.; Ernst, R.; Fitzsimons, I.; Fuck, R.; Gladkochub, D.; Jacobs, J.; et al. Assembly, configuration, and break-up history of Rodinia: A synthesis. Precambrian Res. 2008, 160, 179-210. [CrossRef]

36. Zhao, G.C.; Cawood, P.A. Precambrian geology of China. Precambrian Res. 2012, 222-223. [CrossRef]

37. McLennan, S.M. Rare earth elements in sedimentary rocks; influence of provenance and sedimentary processes. Rev. Mineral. Geochem. 1989, 21, 169-200. [CrossRef]

38. Bau, M.; Dulski, P. Distribution of yttrium and rare-earth elements in the Penge and Kuruman iron-formations, Transvaal Supergroup, South Africa. Precambrian Res. 1996, 79, 37-55. [CrossRef]

39. Hoffman, P.F. Did the Breakout of Laurentia Turn Gondwanaland Inside-Out? Science 1991, 252, 1409-1412. [CrossRef] [PubMed]

40. Kirschvink, J. A Paleogeographic Model for Vendian and Cambrian Time. In The Proterozoic Biosphere: A Multidisciplinary Study; Cambridge University Press: New York, NY, USA, 1992.

41. Zhang, J.; Sun, C.M.; Yang, G.F.; Xie, F. Geochemical characteristics of REE in black shale of lower Cambrian series of Guizhou. Chin. Rare Earths 2008, 29, 72-75, (In Chinese with English abstract). [CrossRef]

42. Wen, H.J.; Carignan, J.; Zhang, Y.X.; Fan, H.F.; Cloquet, C.; Liu, S.R. Molybdenum isotopic records across the Precambrian-Cambrian boundary. Geology 2011, 39, 775-778. [CrossRef]

43. Fleet, M.E.; Pan, Y. Site preference of rare earth elements in fluorapatite. Am. Mineral. 1995, 80, 329-335. [CrossRef]

44. Hughes, J.M.; Rakovan, J. The Crystal Structure of Apatite, Ca5(PO4)3(F,OH,Cl). Rev. Miner. Geochem. 2002, 48, 1-12. [CrossRef] 
45. Pan, Y.; Fleet, M.E. Compositions of the Apatite-Group Minerals: Substitution Mechanisms and Controlling Factors. Rev. Miner. Geochem. 2002, 48, 13-49. [CrossRef]

46. Mercer, C.N.; Watts, K.E.; Gross, J. Apatite trace element geochemistry and cathodoluminescent textures-A comparison between regional Magmatism and the Pea Ridge IOAREE and Boss IOCG deposits, southeastern Missouri iron metallogenic province, USA. Ore Geol. Rev. 2020, 116, 103129. [CrossRef]

47. Goldoff, B.; Webster, J.D.; Harlov, D.E. Characterization of fluor-chlorapatites by electron probe microanalysis with a focus on time-dependent intensity variation of halogens. Am. Miner. 2012, 97, 1103-1115. [CrossRef]

48. Harlov, D.E.; Meighan, C.J.; Kerr, I.D.; Samson, I.M. Mineralogy, Chemistry, and Fluid-Aided Evolution of the Pea Ridge Fe Oxide-(Y + REE) Deposit, Southeast Missouri, USA. Econ. Geol. 2016, 111, 1963-1984. [CrossRef]

49. Liu, Y.; Hu, Z.; Zong, K.; Gao, C.; Gao, S.; Xu, J.; Chen, H. Reappraisement and refinement of zircon U-Pb isotope and trace element analyses by LA-ICP-MS. Chin. Sci. Bull. 2010, 55, 1535-1546. [CrossRef]

50. Liu, Y.; Hu, Z.; Gao, S.; Günther, D.; Xu, J.; Gao, C.; Chen, H. In situ analysis of Major and trace elements of anhydrous minerals by LA-ICP-MS without applying an internal standard. Chem. Geol. 2008, 257, 34-43. [CrossRef]

51. Taylor, S.R.; McLennan, S.M. The Continental Crust: Its Composition and Evolution; Blackwell Scientific Publication: Oxford, UK, 1985; p. 312.

52. Alibo, D.S.; Nozaki, Y. Rare earth elements in seawater: Particle association, shale-normalization, and Ce oxidation. Geochim. Cosmochim. Acta 1999, 63, 363-372. [CrossRef]

53. Liao, J.; Sun, X.; Li, D.; Sa, R.; Lu, Y.; Lin, Z.; Xu, L.; Zhan, R.; Pan, Y.; Xu, H. New insights into nanostructure and geochemistry of bioapatite in REE-rich deep-sea sediments: LA-ICP-MS, TEM, and Z-contrast imaging studies. Chem. Geol. 2019, 512, 58-68. [CrossRef]

54. Auer, G.; Reuter, M.; Hauzenberger, C.A.; Piller, W.E. The impact of transport processes on rare earth element patterns in Marine authigenic and biogenic phosphates. Geochim. Cosmochim. Acta 2017, 203, 140-156. [CrossRef]

55. Liao, J.; Sun, X.; Wu, Z.; Sa, R.; Guan, Y.; Lu, Y.; Li, D.; Liu, Y.; Deng, Y.; Pan, Y. Fe-Mn (oxyhydr)oxides as an indicator of REY enrichment in deep-sea sediments from the central North Pacific. Ore Geol. Rev. 2019, 112, 103044. [CrossRef]

56. Takahashi, Y.; Manceau, A.; Geoffroy, N.; Marcus, M.A.; Usui, A. Chemical and structural control of the partitioning of $\mathrm{Co}, \mathrm{Ce}$, and $\mathrm{Pb}$ in Marine ferromanganese oxides. Geochim. Cosmochim. Acta 2007, 71, 984-1008. [CrossRef]

57. Onuma, N.; Higuchi, H.; Wakita, H.; Nagasawa, H. Trace element partition between two pyroxenes and the host lava. Earth Planet. Sci. Lett. 1968, 5, 47-51. [CrossRef]

58. Zeng, L.-J.; Niu, H.-C.; Yang, W.; Bao, Z.-W. Chemical lattice expansion of natural zircon during the Magmatic-hydrothermal evolution of A-type granite. Am. Miner. 2017, 102, 655-665. [CrossRef]

59. Blundy, J.D.; Wood, B. Prediction of crystal-melt partition coefficients from elastic moduli. Nat. Cell Biol. 1994, 372, 452-454. [CrossRef]

60. German, C.R.; Elderfield, H. Application of the Ce anomaly as a paleoredox indicator: The ground rules. Paleoceanogr. Paleocl. 1990, 5, 823-833. [CrossRef]

61. Elderfield, H. The oceanic chemistry of the rare-earth elements. Philos. Trans. R. Soc. Lond. Ser. A 1988, 325, 105-126. [CrossRef]

62. Morad, S.; Felitsyn, S. Identification of primary Ce-anomaly signatures in fossil biogenic apatite: Implication for the Cambrian oceanic anoxia and phosphogenesis. Sediment. Geol. 2001, 143, 259-264. [CrossRef]

63. Wright, J.; Seymour, R.S.; Shaw, H.F. REE and Nd isotopes in conodont apatite: Variations with geological age and depositional environment. In Special Paper of the Geological Society of America; Geological Society of America: Boulder, CO, USA, 1984; Volume 196, pp. 325-340.

64. Nozaki, Y.; Zhang, J.; Amakawa, H. The fractionation between $\mathrm{Y}$ and Ho in the Marine environment. Earth Planet. Sci. Lett. 1997, 148, 329-340. [CrossRef]

65. Bau, M.; Möller, P.; Dulski, P. Yttrium and lanthanides in eastern Mediterranean seawater and their fractionation during redox-cycling. Mar. Chem. 1997, 56, 123-131. [CrossRef]

66. Olivarez, A.M.; Owen, R.M. The europium anomaly of seawater: Implications for fluvial versus hydrothermal REE inputs to the oceans. Chem. Geol. 1991, 92, 317-328. [CrossRef]

67. Fan, H.; Wen, H.; Zhu, X.; Hu, R.; Tian, S. Hydrothermal activity during Ediacaran-Cambrian transition: Silicon isotopic evidence. Precambrian Res. 2013, 224, 23-35. [CrossRef] 
68. Michard, A.; Gurriet, P.; Soudant, M.; Albarede, F. Nd isotopes in French Phanerozoic shales: External vs. internal aspects of crustal evolution. Geochim. Cosmochim. Acta 1985, 49, 601-610. [CrossRef]

69. Berner, R.A. Phosphate removal from sea water by adsorption on volcanogenic ferric oxides. Earth Planet. Sci. Lett. 1973, 18, 77-86. [CrossRef]

70. Poulton, S.W.; Canfield, D.E. Co-diagenesis of iron and phosphorus in hydrothermal sediments from the southern East Pacific Rise: Implications for the evaluation of paleoseawater phosphate concentrations. Geochim. Cosmochim. Acta 2006, 70, 5883-5898. [CrossRef]

Publisher's Note: MDPI stays neutral with regard to jurisdictional claims in published maps and institutional affiliations.

(C) 2020 by the authors. Licensee MDPI, Basel, Switzerland. This article is an open access article distributed under the terms and conditions of the Creative Commons Attribution (CC BY) license (http://creativecommons.org/licenses/by/4.0/). 